

\title{
Surpassing the ratios conjecture in the 1-level density of Dirichlet $L$-functions
}

\author{
Daniel Fiorilli and Steven J. Miller
}

We study the 1-level density of low-lying zeros of Dirichlet $L$-functions in the family of all characters modulo $q$, with $Q / 2<q \leq Q$. For test functions whose Fourier transform is supported in $\left(-\frac{3}{2}, \frac{3}{2}\right)$, we calculate this quantity beyond the square root cancellation expansion arising from the $L$-function ratios conjecture of Conrey, Farmer and Zirnbauer. We discover the existence of a new lower-order term which is not predicted by this powerful conjecture. This is the first family where the 1-level density is determined well enough to see a term which is not predicted by the ratios conjecture, and proves that the exponent of the error term $Q^{-1 / 2+\epsilon}$ in the ratios conjecture is best possible. We also give more precise results when the support of the Fourier transform of the test function is restricted to the interval $[-1,1]$. Finally we show how natural conjectures on the distribution of primes in arithmetic progressions allow one to extend the support. The most powerful conjecture is Montgomery's, which implies that the ratios conjecture's prediction holds for any finite support up to an error $Q^{-1 / 2+\epsilon}$.

1. Introduction 13

2. Background and new results 18

3. The explicit formula and needed sums 26

4. Unconditional results (Theorems 2.1 and 2.3) 37

5. Results under GRH (Theorems 1.2 and 2.6) 39

6. Results under de-averaging hypothesis (Theorem 2.8) 45

7. Results under Montgomery's hypothesis (Theorem 2.13) 47

Acknowledgments $\quad 48$

References $\quad 48$

\section{Introduction}

In this paper we study the 1-level density of Dirichlet $L$-functions with modulus $q$. The goal is to compute this statistic for large support and small error terms, providing

MSC2010: primary 11M26, 11M50, 11N13; secondary 11N56, $15 \mathrm{~B} 52$.

Keywords: low-lying zeros, Dirichlet L-functions, ratios conjecture, primes in arithmetic progressions, random matrix theory. 
a test of the predictions of the lower order and error terms in the $L$-function ratios conjecture. In this introduction we assume the reader is familiar with low-lying zeros of families of $L$-functions and the ratios conjecture, and briefly describe our results. For completeness we provide a brief review of the subject in Section 2A, and state our results in full in Section 2B to Section 2D.

We let $\eta \in L^{1}(\mathbb{R})$ be an even real function such that $\hat{\eta}$ is $C^{2}$ and has compact support. Denoting by $\rho_{\chi}=\frac{1}{2}+i \gamma_{\chi}$ the nontrivial zeros of $L(s, \chi)$ (i.e., those satisfying $\left.0<\Re\left(\rho_{\chi}\right)<1\right)$ and choosing $Q$ a scaling parameter close to $q$, the 1 -level density is ${ }^{1}$

$$
D_{1 ; q}(\eta):=\frac{1}{\phi(q)} \sum_{\chi \bmod q} \sum_{\gamma_{\chi}} \eta\left(\gamma_{\chi} \frac{\log Q}{2 \pi}\right) .
$$

Throughout this paper, a sum over $\chi \bmod q$ always means a sum over all characters, including the principal character. If we assume GRH then the $\gamma_{\chi}$ are real. As $\eta(y)=\widehat{(\hat{\eta})}(y)$ is defined for complex values of $y$, it makes sense to consider (1-1) for complex $\gamma_{\chi}$, in case GRH is false (in other words, GRH is only needed to interpret the 1-level density as a spacing statistic arising from an ordered sequence of real numbers, allowing for a spectral interpretation). We also study the average of (1-1) over the moduli $Q / 2<q \leq Q$, which is easier to understand in general:

$$
D_{1 ; Q / 2, Q}(\eta):=\frac{1}{Q / 2} \sum_{Q / 2<q \leq Q} D_{1 ; q}(\eta) .
$$

The powerful ratios conjecture of Conrey, Farmer and Zirnbauer [Conrey et al. 2008; Conrey et al. 2005b] yields a formula for $D_{1 ; Q / 2, Q}(\eta)$, which is believed to hold up to an error of $O_{\epsilon}\left(Q^{-1 / 2+\epsilon}\right)$. While there have been several papers [Conrey and Snaith 2007; 2008; David et al. 2013; Goes et al. 2010; Huynh et al. 2011; Miller 2008; 2009b; Miller and Montague 2011] showing agreement between various statistics involving $L$-functions and the ratios conjecture's predictions, evidence for this precise exponent in the error term is limited; the reason this exponent was chosen is the "philosophy of square root cancellation". While some of the families studied have 1-level densities that agree beyond square root cancellation, it is always for small support $\operatorname{supp}(\widehat{\eta}) \subset(-1,1))$. Further, in no family studied were nonzero lower order terms beyond square root cancellation isolated in the 1-level density.

The motivation of this paper was to resolve these issues. As the ratios conjecture is used in a variety of problems, it is important to test its predictions in the greatest possible window. Our key findings are the following.

\footnotetext{
${ }^{1}$ Since $\hat{\eta}$ is $C^{2}$, we have $\eta(\xi) \ll \xi^{-2}$ for large $\xi$; hence the sum over the zeros is absolutely convergent. While most of the literature uses $\phi$ as the test function, since we will use Euler's totient function extensively we use $\eta$.
} 
- We uncover new, nonzero lower-order terms in the 1-level density for our families of Dirichlet characters. These terms are beyond what the ratios conjecture can predict, and suggest the possibility that a refinement may be possible and needed.

- We show (unconditionally) that the natural limit of accuracy of the $L$-function ratios conjecture is $\Omega\left(Q^{-1 / 2+o(1)}\right)$. Thus the error term cannot be improved for a general family of $L$-functions, though of course its veracity for all families is still open.

The existence of lower-order terms beyond the ratios conjecture's prediction in statistics of $L$-functions is not without precedent. Indeed such terms have been isolated in the second moment of $\left|L\left(\frac{1}{2}, \chi\right)\right|$ by Heath-Brown [1981], and for a more general shifted sum by Conrey [2007].

Before stating our main result, we give the ratios conjecture's prediction. This prediction is done for a slightly different family in [Goes et al. 2010], but it is trivial to convert from their formulation to the one below (we discuss the conversion in Section 2B).

Conjecture 1.1 (ratios conjecture). The 1-level density $D_{1 ; q}(\eta)$ (from (1-1) with scaling parameter $Q=q$ ) equals

$$
\begin{aligned}
\hat{\eta}(0)\left(1-\frac{\log \left(8 \pi e^{\gamma}\right)}{\log q}-\frac{1}{\log q} \sum_{p \mid q}\right. & \left.\frac{\log p}{p-1}\right) \\
& +\int_{0}^{\infty} \frac{\hat{\eta}(0)-\hat{\eta}(t)}{q^{t / 2}-q^{-t / 2}} d t+O_{\epsilon}\left(q^{-1 / 2+\epsilon}\right) .
\end{aligned}
$$

The 1-level density $D_{1 ; Q / 2, Q}(\eta)$ (from rescaling ${ }^{2}(1-3)$ ) equals

$$
\begin{aligned}
\hat{\eta}(0)\left(1-\frac{\log \left(4 \pi e^{\gamma}\right)+1}{\log Q}-\frac{1}{\log Q} \sum_{p} \frac{\log p}{p(p-1)}\right) \\
+\int_{0}^{\infty} \frac{\hat{\eta}(0)-\hat{\eta}(t)}{Q^{t / 2}-Q^{-t / 2}} d t+O_{\epsilon}\left(Q^{-1 / 2+\epsilon}\right) .
\end{aligned}
$$

Surprisingly, our techniques are capable of not only verifying this prediction, but we are able to determine the 1-level density beyond what even the ratios conjecture predicts. In Theorem 1.2 we obtain a new (arithmetical) term of order $Q^{-1 / 2} / \log Q$, which is not predicted by the ratios conjecture.

${ }^{2}$ To rescale we multiply (1-3) by $\log q / \log Q$, replace $q^{t / 2}-q^{-t / 2}$ with $Q^{t / 2}-Q^{-t / 2}$ and average over $Q / 2<q \leq Q$. The term $\log q$ averages to $\log Q+\log 2-1+O(\log Q / Q)$, explaining the "additional" term $(\log 2-1) / \log Q$. Moreover the average of $\sum_{p \mid q} \frac{\log p}{p-1}$ over this range is easily shown to be $\sum_{p} \frac{1}{p(p-1)}+O(\log Q / Q)$. 
Theorem 1.2. Assume GRH. If the Fourier transform of the test function $\eta$ is supported in $\left(-\frac{3}{2}, \frac{3}{2}\right)$, then $D_{1 ; q / 2, Q}(\eta)$ equals

$$
\begin{aligned}
\hat{\eta}(0)\left(1-\frac{\log \left(4 \pi e^{\gamma}\right)+1}{\log Q}-\frac{1}{\log Q} \sum_{p} \frac{\log p}{p(p-1)}\right) \\
+\int_{0}^{\infty} \frac{\hat{\eta}(0)-\hat{\eta}(t)}{Q^{t / 2}-Q^{-t / 2}} d t+\frac{Q^{-1 / 2}}{\log Q} S_{\eta}(Q),
\end{aligned}
$$

where

$$
S_{\eta}(Q)=C_{1} \hat{\eta}(1)+C_{2} \frac{\hat{\eta}^{\prime}(1)}{\log Q}+O\left(\left(\frac{\log \log Q}{\log Q}\right)^{2}\right)
$$

with

$$
\begin{aligned}
& C_{1}:=(2-\sqrt{2}) \zeta\left(\frac{1}{2}\right) \prod_{p}\left(1+\frac{1}{(p-1) p^{1 / 2}}\right), \\
& C_{2}:=C_{1}\left(\frac{\sqrt{2}+4}{3}-\left(\frac{\zeta^{\prime}}{\zeta}\left(\frac{1}{2}\right)-\sum_{p} \frac{\log p}{(p-1) p^{1 / 2}+1}\right)\right) .
\end{aligned}
$$

We can give a more precise formula for the term $S_{\eta}(Q)$ : see Remark 2.5. While Theorem 1.2 is conditional on GRH, in Theorem 2.1 we prove a more precise and unconditional result for test functions $\eta$ whose Fourier transform has support contained in $[-1,1]$.

The first two terms in (1-5) agree with the ratios conjecture's prediction. As for the term $Q^{-1 / 2} S_{\eta}(Q) / \log Q$, its presence confirms that the error term $Q^{-1 / 2+o(1)}$ in the ratios conjecture is best possible, and suggests more generally that the 1-level density of a family ought to contain a (possibly oscillating) arithmetical term of order $Q^{-1 / 2+o(1)}$, a statement which should be tested in other families. Interestingly this new term contains the factors $\hat{\eta}(1)$ and $\hat{\eta}^{\prime}(1)$, and is zero when $\hat{\eta}$ is supported in $(-1,1)$. In this case we give a more precise estimate for the 1-level density in Theorem 2.1, in which a lower-order term of order $Q^{\sigma / 2-1+o(1)}$ appears, where $\sigma=\sup (\operatorname{supp} \hat{\eta})$. This term is a genuine lower-order term, and shows that for such test functions the ratios conjecture's prediction is not best possible. We thus show that a transition happens when $\sigma$ is near 1. Indeed looking at the difference between the 1-level density and the ratios conjecture's prediction, that is defining

$$
\begin{aligned}
E_{Q}(\eta):=D_{1 ; Q / 2, Q}(\eta)-\hat{\eta}(0)\left(1-\frac{\log \left(4 \pi e^{\gamma}\right)+1}{\log Q}\right. & \left.-\frac{1}{\log Q} \sum_{p} \frac{\log p}{p(p-1)}\right) \\
& -\int_{0}^{\infty} \frac{\hat{\eta}(0)-\hat{\eta}(t)}{Q^{t / 2}-Q^{-t / 2}} d t,
\end{aligned}
$$


our results imply that ${ }^{3} E_{Q}(\eta)=Q^{-\mu(\sigma)+o(1)}$, where

$$
\mu(\sigma)= \begin{cases}\frac{\sigma}{2}-1 & \text { if } \sigma \leq 1, \\ -\frac{1}{2} & \text { if } 1 \leq \sigma<\frac{3}{2}\end{cases}
$$

We conjecture that $\mu(\sigma)$ should equal $-\frac{1}{2}$ for all $\sigma \geq 1$, and that our new lower-order term $Q^{-1 / 2} S_{\eta}(Q) / \log Q$ should persist in this extended range.

Conjecture 1.3. Theorem 1.2 holds for test functions $\eta$ whose Fourier transform has arbitrarily large finite support $\sigma$.

In Figure 1, the solid curve represents our results (Theorems 1.2 and 2.1), and the dashed line represents Conjecture 1.3; note the resemblance between this graph and the one appearing in Montgomery's pair correlation conjecture [Montgomery 1973]. We prove in Theorem 2.13 that Montgomery's conjecture on primes in arithmetic progressions implies that $\mu(\sigma) \leq-\frac{1}{2}$ for all $\sigma \geq 1$.

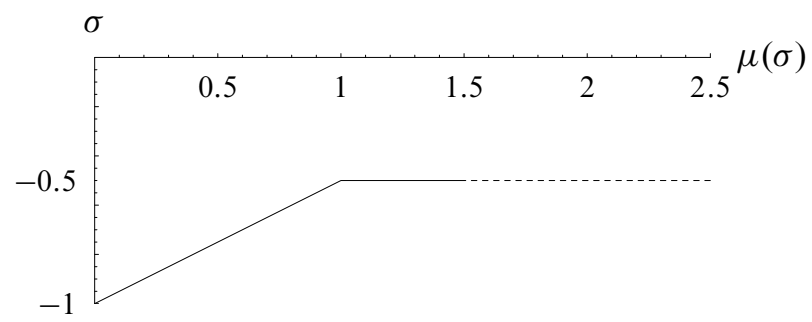

Figure 1. The graph of $\mu(\sigma)$.

We believe that this phenomenon is universal and should also happen in different families, in the sense that we believe that the ratios conjecture's prediction should be best possible for $\sigma \geq 1$, and should not be for $\sigma<1$. For example, in [Miller $2009 \mathrm{~b}$ ] it is shown that if the Fourier transform of the involved test function is supported in $(-1,1)$, then the ratios conjecture's prediction is not best possible and one can improve the remainder term; however, in this region of limited support there are no new, nonzero lower order terms unpredicted by the ratios conjecture. These results confirm the exceptional nature of the transition point $\sigma=1$, as is the case in Montgomery's pair correlation conjecture [1973]. Indeed if this last conjecture were known to hold beyond the point $\alpha=1$, then this would imply the nonexistence of Landau-Siegel zeros [Conrey and Iwaniec 2002].

Our plan of attack is to use the explicit formula to turn the 1-level density into an average of the various terms appearing in this formula. The bulk of the work is devoted to carefully estimating the contribution of the prime sum, which when summing over $\chi \bmod q$ becomes a sum over primes in the residue class $1 \bmod q$,

\footnotetext{
${ }^{3}$ For $\sigma>1$, this holds for test functions $\eta$ for which either $\hat{\eta}(1) \neq 0$ or $\hat{\eta}^{\prime}(1) \neq 0$ (see Theorem 1.2); see Theorem 2.1 if $\sigma \leq 1$. If $\hat{\eta}(u)$ vanishes in a small interval around $u=1$, then Theorem 2.6 gives the correct answer.
} 
averaged over $q \sim Q$. Accordingly, the proof of Theorem 1.2 is based on ideas in [Fiorilli 2012], which improve on results of Fouvry [1985], Bombieri, Friedlander and Iwaniec [Bombieri et al. 1986], Friedlander and Granville [1992] and Friedlander, Granville, Hildebrandt and Maier [Friedlander et al. 1991]. Theorem 1.1 of [Fiorilli 2012] cannot be applied directly here, since this estimate is only valid when looking at primes up to $x$ modulo $q$ with $q \sim Q$, where $Q$ is not too close to $x$. Additional estimates are needed, including a careful analysis of the range $x^{1-\epsilon}<Q \leq x$, which required a combination of divisor switching techniques and precise estimates on the mean value of smoothed sums of the reciprocal of Euler's totient function. Additionally, in our analysis of the 1-level density after using the explicit formula and executing the sum over the family we obtain a sum over primes in the arithmetic progressions $1 \bmod q$; this is one of the cases where one obtains an asymptotic in [Fiorilli 2012, Theorem 1.1], which explains the occurrence of the lower-order term $Q^{-1 / 2} S_{\eta}(Q) / \log Q$ in Theorem 1.2.

The paper is organized as follows. In Section $2 \mathrm{~A}$ we review previous results on low-lying zeros in families of $L$-functions and describe the motivation for the ratios conjecture. See for example [Goes et al. 2010; Miller 2009b] for a detailed description of how to apply the ratios conjecture to predict the 1-level density. We describe our unconditional results in Section $2 \mathrm{~B}$, and then improve our results in Section $2 \mathrm{C}$ by assuming GRH. In previous families there often is a natural barrier, and extending the support is related to standard conjectures (for example, in [Iwaniec et al. 2000] the authors show how cancellation in exponential sums involving square roots of primes leads to larger support for families of cuspidal newforms). A similar phenomenon surfaces here, where in Section 2D we show that increasing the support beyond $(-2,2)$ is related to conjectures on the distribution of primes in residue classes. We analyze the increase in support provided by various conjectures. These range from a conjecture on the variance of primes in the residue classes, which allow us to reach $(-4,4)$, to Montgomery's conjecture for a fixed residue, which gives us any finite support. The next sections contain the details of the proof; we state the explicit formula and prove some needed sums in Section 3, and then prove our theorems in the remaining sections.

\section{Background and new results}

2A. Background and previous results. Assuming GRH, the nontrivial zeros of any nice $L$-function lie on the critical line, and therefore it is possible to investigate statistics of its normalized zeros. These zeros are fundamental in many problems, ranging from the distribution of primes in congruence classes to the class number [Conrey and Iwaniec 2002; Goldfeld 1976; Gross and Zagier 1986; Rubinstein and Sarnak 1994]. Numerical and theoretical evidence [Hejhal 1994; Montgomery 
1973; Odlyzko 1987; 2001; Rudnick and Sarnak 1996] support a universality in behavior of zeros of an individual automorphic $L$-function high above the central point, specifically that they are well-modeled by ensembles of random matrices (see [Firk and Miller 2009; Hayes 2003] for histories of the emergence of random matrix theory in number theory). The story is different for the low-lying zeros, the zeros near the central point. A convenient way to study these zeros is via the 1-level density, which we now describe. Let $\eta \in L^{1}(\mathbb{R})$ be an even real function whose Fourier transform

$$
\hat{\eta}(y)=\int_{-\infty}^{\infty} \eta(x) e^{-2 \pi i x y} d x
$$

is $C^{2}$ and has compact support. Let $\mathscr{F}_{N}$ be a (finite) family of $L$-functions satisfying GRH. ${ }^{4}$ The 1 -level density associated to $\mathscr{F}_{N}$ is defined by

$$
D_{1 ; \mathscr{F}_{N}}(\eta)=\frac{1}{\left|\mathscr{F}_{N}\right|} \sum_{g \in \mathscr{F}_{N}} \sum_{j} \eta\left(\frac{\log c_{g}}{2 \pi} \gamma_{g}^{(j)}\right),
$$

where $\frac{1}{2}+i \gamma_{g}^{(j)}$ runs through the nontrivial zeros of $L(s, g)$. Here $c_{g}$ is the "analytic conductor" of $g$, and gives the natural scale for the low zeros. As $\eta$ decays, only low-lying zeros (i.e., zeros within a distance $1 / \log c_{g}$ of the central point $s=\frac{1}{2}$ ) contribute significantly. Thus the 1-level density can help identify the symmetry type of the family. To evaluate (2-2), one applies the explicit formula, converting sums over zeros to sums over primes.

Based in part on the function field analysis where $G(\mathscr{F})$ is the monodromy group associated to the family $\mathscr{F}$, Katz and Sarnak conjectured that for each reasonable irreducible family of $L$-functions there is an associated symmetry group $G(\mathscr{F})$ (one of the following five: unitary U, symplectic USp, orthogonal O, SO(even), $\mathrm{SO}$ (odd)), and that the distribution of critical zeros near $\frac{1}{2}$ mirrors the distribution of eigenvalues near 1 . The five groups have distinguishable 1-level densities. To date, for suitably restricted test functions the statistics of zeros of many natural families of $L$-functions have been shown to agree with statistics of eigenvalues of matrices from the classical compact groups, including Dirichlet $L$-functions, elliptic curves, cuspidal newforms, Maass forms, number field $L$-functions, and symmetric powers of $\mathrm{GL}_{2}$ automorphic representations [Alpoge and Miller 2014; Alpoge et al. 2014; Dueñez and Miller 2006; Fouvry and Iwaniec 2003; Gao 2005; Güloğlu 2005; Hughes and Miller 2007; Hughes and Rudnick 2003; Iwaniec et al. 2000; Katz and Sarnak 1999a; 1999b; Miller 2004; Miller and Peckner 2012; Ricotta and Royer 2011; Royer 2001; Rubinstein 2001; Shin and Templier 2012; Yang 2009; Young

${ }^{4}$ We often do not need GRH for the analysis, but only to interpret the results. If the GRH is true, the zeros lie on the critical line and can be ordered, which suggests the possibility of a spectral interpretation. 
2006], to name a few, as well as nonsimple families formed by Rankin-Selberg convolution [Dueñez and Miller 2009].

In addition to predicting the main term (see for example [Conrey 2001; Katz and Sarnak 1999a; 1999b; Keating and Snaith 2000a; 2000b; 2003]), techniques from random matrix theory have led to models that capture the lower order terms in their full arithmetic glory for many families of $L$-functions (see for example the moment conjectures in [Conrey et al. 2005a] or the hybrid model in [Gonek et al. 2007]). Since the main terms agree with either unitary, symplectic or orthogonal symmetry, it is only in the lower order terms that we can break this universality and see the arithmetic of the family enter. These are therefore natural and important objects to study, and can be isolated in many families [Huynh et al. 2009; Miller 2009a; Young 2005]. We thus require a theory that is capable of making detailed predictions. Recently the $L$-function ratios conjecture [Conrey et al. 2008; 2005b] has had great success in determining lower order terms. Though a proof of the ratios conjecture for arbitrary support is well beyond the reach of current methods, it is an indispensable tool in current investigations as it allows us to easily write down the predicted answer to a remarkable level of precision, which we try to prove in as great a generality as possible.

To study the 1-level density, it suffices to obtain good estimates for

$$
R_{\mathscr{F}_{N}}(\alpha, \gamma):=\frac{1}{\left|\mathscr{F}_{N}\right|} \sum_{g \in \mathscr{F}_{N}} \frac{L\left(\frac{1}{2}+\alpha, g\right)}{L\left(\frac{1}{2}+\gamma, g\right)}
$$

(In the current paper, the parameter $Q$ plays the role of $\left|\mathscr{F}_{N}\right|$.) Asymptotic formulas for $R_{\mathscr{F}_{N}}(\alpha, \gamma)$ have been conjectured for a variety of families $\mathscr{F}_{N}$ (see [Conrey et al. 2008; Conrey and Snaith 2007; 2008; Goes et al. 2010; Huynh et al. 2011; Miller 2008; 2009b; Miller and Montague 2011]) and are believed to hold up to errors of size $\left|\mathscr{F}_{N}\right|^{-1 / 2+\epsilon}$ for any $\epsilon>0$. The evidence for the correctness of this error term is limited to test functions with small support (frequently significantly less than $(-1,1))$, though in such regimes many of the above papers verify this prediction. Many of the steps in the ratios conjecture's recipe lead to the addition or omission of terms as large as those being considered, and thus there was uncertainty as to whether or not the resulting predictions should be accurate to square root cancellation. The results of the current paper can be seen as a confirmation that this is the right error term for the final predicted answer, at least in this family. Further, the novelty in our results resides in the fact that we are able to go beyond square root cancellation and we find a smaller term which is unpredicted by the ratios conjecture (see Theorem 1.2). For a precise explanation on how to derive the ratios conjecture's prediction in our family, we refer the reader to [Goes et al. 2010], and also recommend [Conrey and Snaith 2007] for an accessible overview of the ratios conjecture. 
2B. Unconditional results. We now describe our unconditional results. We remind the reader that $\eta$ is a real even function such that $\hat{\eta}$ is $C^{2}$ and has compact support.

Theorem 2.1. Suppose that the Fourier transform of the test function $\eta$ is supported on the interval $[-1,1]$, so $\sigma=\sup (\operatorname{supp} \hat{\eta}) \leq 1$. There exists an absolute positive constant c (coming from the Prime Number Theorem) such that the 1-level density $D_{1 ; q}(\eta)$ (from (1-1) with scaling parameter $Q=q$ ) equals

$$
\begin{gathered}
\hat{\eta}(0)\left(1-i \frac{\log \left(8 \pi e^{\gamma}\right)}{\log q}-\frac{1}{\log q} \sum_{p \mid q} \frac{\log p}{p-1}\right) \\
+\int_{0}^{\infty} \frac{\hat{\eta}(0)-\hat{\eta}(t)}{q^{t / 2}-q^{-t / 2}} d t-\frac{2}{\phi(q)} \int_{0}^{1} q^{u / 2}\left(\frac{\hat{\eta}(u)}{2}-\frac{\hat{\eta}^{\prime}(u)}{\log q}\right) d u \\
-\frac{2}{\log q} \sum_{\substack{p^{v} \| q \\
p^{e} \equiv 1 \bmod q / p^{\nu} \\
e, \nu \geq 1}} \frac{\log p}{\phi\left(p^{v}\right) p^{e / 2}} \hat{\eta}\left(\frac{\log p^{e}}{\log q}\right)+O\left(\frac{q^{\sigma / 2-1}}{e^{c \sqrt{\sigma \log q}}}\right) .
\end{gathered}
$$

Remark 2.2. The average over $Q / 2<q \leq Q$ of the fourth term in (2-4) can be shown to be $O\left(Q^{-1}\right)$, and is therefore negligible when considering $D_{1 ; Q / 2, Q}(\eta)$ (see (3-16)). However, the term involving the second integral in (2-4) is of size $q^{\sigma / 2-1-o(1)}$, and thus constitutes a genuine lower-order term, smaller than the error term in (1-3) predicted using the ratios conjecture.

Theorems 1.2 and 2.1 should be compared to the main result of Goes, Jackson, Miller, Montague, Ninsuwan, Peckner and Pham [Goes et al. 2010], where they show one can extend the support of $\hat{\eta}$ to $[-2,2]$ and still get the main term, as well as the lower order terms down to a power savings. They only consider $q$ prime, and thus the sum over primes $p$ dividing $q$ below in Theorem 2.3 is absorbed by their error term. We briefly discuss how one can easily extend their results to the case of general $q$. First note that $L(s, \chi)$ and $L\left(s, \chi^{*}\right)$ have the same zeros in the critical strip if $\chi^{*}$ is the primitive character of conductor $q^{*}$ inducing the nonprincipal character $\chi$ of conductor $q$. We now have $\log q^{*}$, which can be converted to a sum over primes $p$ dividing $q$ by the same arguments as in the proof of Proposition 3.1. The rest of the expansion follows from expanding the digamma function $\Gamma^{\prime} / \Gamma$ in the integral in [Goes et al. 2010, Theorem 1.3] and then standard algebra (along the lines of the computations in Section 3). We use [Montgomery and Vaughan 2007, Lemma 12.14], which in our notation says that for $a, b>0$ we have

$$
\begin{aligned}
& \int_{-\infty}^{\infty} \frac{\Gamma^{\prime}(a \pm i b \tau)}{\Gamma(a \pm i b \tau)} \eta(t) d t \\
& \quad=\frac{\Gamma^{\prime}(a)}{\Gamma(a)} \hat{\eta}(0)+\frac{2 \pi}{b} \int_{0}^{\infty} \frac{\exp (-2 \pi a x / b)}{1-\exp (-2 \pi x / b)}(\widehat{\eta}(0)-\hat{\eta}(\mp x)) d x,
\end{aligned}
$$


and the identity

$$
\frac{\Gamma^{\prime}\left(\frac{1}{4}\right)}{\Gamma\left(\frac{1}{4}\right)}+\frac{\Gamma^{\prime}\left(\frac{3}{4}\right)}{\Gamma\left(\frac{3}{4}\right)}=-2 \gamma-6 \log 2,
$$

with $\gamma$ the Euler-Mascheroni constant. We then extend to $q \in(Q / 2, Q]$ by rescaling the zeros by $\log Q$ and not $\log q$ and summing over the family; recall the technical issues involved in the rescaling are discussed in Footnote 2.

Theorem 2.3 (Goes, Jackson, Miller, Montague, Ninsuwan, Peckner, Pham [Goes et al. 2010]). If $1<\sigma \leq 2$, then the 1-level density $D_{1 ; q}(\eta)$ (from (1-1) with scaling parameter $Q=q$ ) equals

$$
\begin{aligned}
\hat{\eta}(0)\left(1-\frac{\log \left(8 \pi e^{\gamma}\right)}{\log q}-\frac{1}{\log q} \sum_{p \mid q} \frac{\log p}{p-1}\right)+\int_{0}^{\infty} \frac{\hat{\eta}(0)-\hat{\eta}(t)}{q^{t / 2}-q^{-t / 2} d t} \\
+O\left(\frac{\log \log q}{\log q} q^{\sigma / 2-1}\right),
\end{aligned}
$$

and this agrees with the ratios conjecture.

Remark 2.4. Goes et al. [2010] actually proved (2-7) for any $\sigma \leq 2$, with the additional error term $O\left(q^{-1 / 2+\epsilon}\right)$. We preferred not to include the case $\sigma \leq 1$, as Theorem 2.1 is more precise in this range.

2C. Results under GRH. We first mention a more precise version of Theorem 1.2.

Remark 2.5. If in addition to the hypotheses of Theorem 1.2 we assume that the Fourier transform of the test function $\eta$ is $K+1$ times continuously differentiable, then we can give a more precise expression for the term $S_{\eta}(Q)$ appearing in (1-5):

$$
S_{\eta}(Q)=\sum_{i=0}^{K} \frac{a_{i}(\eta)}{(\log Q)^{i}}+O_{\epsilon, K}\left(\frac{1}{(\log Q)^{K+1-\epsilon}}\right),
$$

where the $a_{i}(\eta)$ are constants depending (linearly) on the Taylor coefficients of $\hat{\eta}(t)$ at $t=1$. In fact, $S_{\eta}(Q)$ is a truncated linear functional, which composed with the Fourier transform operator is supported on $\{1\}$ (in the sense of distributions).

Our next result is an extension of Theorem 1.2, in the case where $\hat{\eta}(u)$ vanishes in a small interval to the right of $u=1$.

Theorem 2.6. Assume GRH.

(1) If $\hat{\eta}$ is supported in $\left(-\frac{3}{2},-1-\kappa\right] \cup[-1,1] \cup\left[1+\kappa, \frac{3}{2}\right)$ for some $\kappa>0$, then for any $\epsilon>0$ the average 1-level density $D_{1 ; Q / 2, Q}(\eta)$ equals 


$$
\begin{aligned}
\hat{\eta}(0)\left(1-\frac{1+}{\log \left(4 \pi e^{\gamma}\right)}-\frac{1}{\log Q} \sum_{p} \frac{\log p}{p(p-1)}\right) \\
\quad+\int_{0}^{\infty} \frac{\hat{\eta}(0)-\hat{\eta}(t)}{Q^{t / 2}-Q^{-t / 2}} d t \\
\quad-\frac{4 \log 2 \zeta(2) \zeta(3)}{Q} \frac{\int_{0}^{1} Q^{u / 2}\left(\frac{\hat{\eta}(u)}{2}-\frac{\hat{\eta}^{\prime}(u)}{\log Q}\right) d u}{} \\
-\int_{1+\kappa}^{4 / 3}\left((u-1) \log Q+C_{6}\right) Q^{-u / 2}\left(\frac{\hat{\eta}(u)}{2}-\frac{\hat{\eta}^{\prime}(u)}{\log Q}\right) d u \\
+O_{\epsilon}\left(Q^{-1 / 2-\kappa+\epsilon}+Q^{-2 / 3} \log Q+Q^{\sigma-2} \log Q\right),
\end{aligned}
$$

with $C_{6}:=\log (\pi / 2)+1+\gamma+\sum_{p} \frac{\log p}{p(p-1)}$.

(For $\sigma \geq \frac{4}{3}$, unless $\hat{\eta}(x)$ has some mass near $x=\lambda$ for some $1<\lambda<4-2 \sigma$, the fourth term in (2-9) goes in the error term, and hence (2-9) reduces to (2-10). However, if $1<\sigma<\frac{4}{3}$, it is always a genuine lower-order term.)

(2) If $f$ is supported in $(-2,-a] \cup[-1,1] \cup[a, 2)$ for some $1 \leq a<2$ (if $a=1$, we have the full interval $(-2,2))$, then $D_{1 ; Q / 2, Q}(\eta)$ equals

$$
\begin{aligned}
\hat{\eta}(0)\left(1-\frac{1+\log \left(4 \pi e^{\gamma}\right)}{\log Q}-\frac{1}{\log Q} \sum_{p} \frac{\log p}{p(p-1)}\right) & \\
& +\int_{0}^{\infty} \frac{\hat{\eta}(0)-\hat{\eta}(t)}{Q^{t / 2}-Q^{-t / 2}} d t \\
& -\frac{4 \log 2}{Q} \frac{\zeta(2) \zeta(3)}{\zeta(6)} \int_{0}^{1} Q^{u / 2}\left(\frac{\hat{\eta}(u)}{2}-\frac{\hat{\eta}^{\prime}(u)}{\log Q}\right) d u \\
& +O\left(Q^{-a / 2}+Q^{\sigma-2} \log Q\right) .
\end{aligned}
$$

Unless $a>1$ and $\sigma<\frac{3}{2}$, the third term of (2-10) goes in the error term.

2D. Results beyond GRH. As the GRH is insufficient to compute the 1-level density for test functions supported beyond $[-2,2]$, we explore the consequences of other standard conjectures in number theory involving the distribution of primes among residue classes. Before stating these conjectures, we first set the notation. Let

$$
\begin{gathered}
\psi(x):=\sum_{n \leq x} \Lambda(n), \quad \psi(x, q, a):=\sum_{\substack{n \leq x \\
n \equiv a \bmod q}} \Lambda(n), \\
E(x, q, a):=\psi(x, q, a)-\frac{\psi(x)}{\phi(q)} .
\end{gathered}
$$


If we assume GRH, we have

$$
\psi(x)=x+O\left(x^{1 / 2}(\log x)^{2}\right), \quad E(x, q, a)=O\left(x^{1 / 2}(\log x)^{2}\right) .
$$

Our first result uses GRH and the following de-averaging hypothesis, which depends on a parameter $\delta \in[0,1]$.

Hypothesis 2.7. We have

$$
\sum_{\frac{O}{2}<q \leq Q}\left|\psi(x ; q, 1)-\frac{\psi(x)}{\phi(q)}\right|^{2} \ll Q^{\delta-1} \sum_{\frac{O}{2}<q \leq Q} \sum_{\substack{1 \leq a \leq q \\(a, q)=1}}\left|\psi(x ; q, a)-\frac{\psi(x)}{\phi(q)}\right|^{2} .
$$

This hypothesis is trivially true for $\delta=1$, and while it is unlikely to be true for $\delta=0$, it is reasonable to expect it to hold for any $\delta>0$. What we need is some control over biases of primes congruent to $1 \bmod q$; Hypothesis 2.7 can be interpreted as bounding the average of $|\psi(x ; q, 1)-\psi(x) / \phi(q)|^{2}$ in terms of the average variance. ${ }^{5}$

Under these hypotheses, we show how to extend the support to a wider but still limited range.

Theorem 2.8. Assume GRH and Hypothesis 2.7 for some $\delta \in(0,1)$. The average 1-level density $D_{1 ; Q / 2, Q}(\hat{\eta})$ equals

$$
\begin{array}{r}
\widehat{\eta}(0)\left(1-\frac{1+\log \left(4 \pi e^{\gamma}\right)}{\log Q}-\frac{1}{\log Q} \sum_{p} \frac{\log p}{p(p-1)}\right)+\int_{0}^{\infty} \frac{\hat{\eta}(0)-\hat{\eta}(t)}{Q^{t / 2}-Q^{-t / 2}} d t \\
+O\left(Q^{(\delta-1) / 2}(\log Q)^{3 / 2}+Q^{(\sigma+2 \delta) / 4-1}(\log Q)^{1 / 3}\right)
\end{array}
$$

which is asymptotic to $\hat{\eta}(0)$ provided the support of $\hat{\eta}$ is contained in $(-4+2 \delta, 4-2 \delta)$.

The proof of Theorem 2.8 is given in Section 6. It uses a result of Goldston and Vaughan [1997], which is an improvement of results of Barban, Davenport, Halberstam, Hooley, Montgomery and others.

Remark 2.9. In Theorem 2.8 we study the weighted 1-level density

$$
D_{1 ; Q / 2, Q}(\eta):=\sum_{Q / 2<q \leq Q} \frac{1}{\phi(q)} \sum_{\chi \bmod q} \sum_{\gamma_{\chi}} \eta\left(\gamma_{\chi} \frac{\log Q}{2 \pi}\right),
$$

which is technically easier to study than the unweighted version

$$
D_{1 ; Q / 2, Q}^{\text {unweighted }}(\eta):=\frac{1}{9 / \pi^{2}(Q / 2)^{2}} \sum_{Q / 2<q \leq Q} \sum_{\chi \bmod } \sum_{q} \eta\left(\gamma_{\chi} \frac{\log Q}{2 \pi}\right) .
$$

${ }^{5}$ Note that we only need this de-averaging hypothesis for the special residue class $a=1$. 
This is similar to many other families of $L$-functions, such as cuspidal newforms [Iwaniec et al. 2000; Miller and Montague 2011] and Maass forms [Alpoge et al. 2014; Alpoge and Miller 2014], where the introduction of weights (arising from the Petersson and Kuznetsov trace formulas) facilitates evaluating the arithmetical terms.

Finally, we show how we can determine the 1-level density for arbitrary finite support, under a hypothesis of Montgomery [1970].

Hypothesis 2.10 (Montgomery). For any $a, q$ such that $(a, q)=1$ and $q \leq x$, we have

$$
\psi(x ; q, a)-\frac{\psi(x)}{\phi(q)} \ll_{\epsilon} x^{\epsilon}\left(\frac{x}{q}\right)^{1 / 2} .
$$

It is by gaining some savings in $q$ in the error $E(x, q, a)$ that we can increase the support for families of Dirichlet $L$-functions. The following weaker version of Montgomery's conjecture, which depends on a parameter $\theta \in\left(0, \frac{1}{2}\right]$, also suffices to increase the support beyond $[-2,2]$.

Hypothesis 2.11. For any $a, q$ such that $(a, q)=1$ and $q \leq x$, we have

$$
\psi(x ; q, 1)-\frac{\psi(x)}{\phi(q)} \ll_{\epsilon} \frac{x^{1 / 2+\epsilon}}{q^{\theta}} .
$$

Hypothesis 2.12. Fix $\epsilon>0$. We have for $x^{\epsilon} \leq q \leq \sqrt{x}$ that

$$
\sum_{\substack{n \leq x \\ n \equiv 1 \bmod q}} \Lambda(n)\left(1-\frac{n}{x}\right)-\frac{1}{\phi(q)} \sum_{n \leq x} \Lambda(n)\left(1-\frac{n}{x}\right)=o\left(x^{1 / 2}\right) .
$$

Note that this is a weighted version of $\psi(x ; q, 1)-\psi(x) / \phi(q)$; that is, we added the weight $(1-n / x)$. The reason for this is that it makes the count smoother, and this makes it easier to analyze in general since the Mellin transform of $g(y):=1-y$ in the interval $[0,1]$ is decaying faster in vertical strips than that of $g(y) \equiv 1$.

Amongst the last three hypotheses, Hypothesis 2.12 is the weakest, but it is still sufficient to derive the asymptotic in the 1-level density for test functions with arbitrary large support.

Theorem 2.13. Suppose that the Fourier transform of $\eta$ has arbitrarily large (but compact) support.

(1) If we assume Hypothesis 2.12, the 1-level density $D_{1 ; q}(\eta)$ equals $\hat{\eta}(0)+o(1)$, agreeing with the scaling limit of unitary matrices. 
(2) If we assume Hypothesis 2.11 for some $0<\theta \leq \frac{1}{2}$, then $D_{1 ; q}(\eta)$ equals

$$
\begin{aligned}
\hat{\eta}(0)\left(1-\frac{\log \left(8 \pi e^{\gamma}\right)}{\log q}-\frac{1}{\log q} \sum_{p \mid q} \frac{\log p}{p-1}\right)+\int_{0}^{\infty} \frac{\hat{\eta}(0)-\hat{\eta}(t)}{q^{t / 2}-q^{-t / 2}} d t & \\
& +O_{\epsilon}\left(q^{-\theta+\epsilon}\right) .
\end{aligned}
$$

Remark 2.14. Under GRH, the left-hand side of (2-19) is $O\left(x^{1 / 2} \log q\right)$. Therefore, if we win by more than a logarithm over GRH, then we have the expected asymptotic for the 1-level density for $\hat{\eta}$ of arbitrarily large finite support.

Interestingly, if we assume Montgomery's conjecture (Hypothesis 2.10), then we can take $\theta=\frac{1}{2}$ in (2-20), and doing so we end up precisely with the ratios conjecture's prediction; see (1-3).

We derive the explicit formula for the families of Dirichlet characters in Section 3, as well as some useful estimates for some of the resulting sums. We give the unconditional results in Section 4, Theorems 2.1 and 2.3. The proofs of Theorems 1.2 and 2.6 are conditional on GRH, and use results in [Friedlander and Granville 1992] and [Fiorilli 2012]; we give them in Section 5. We conclude with an analysis of the consequences of the hypotheses on the distribution of primes in residue classes, using the de-averaging hypothesis to prove Theorem 2.8 in Section 6 and Montgomery's hypothesis to prove Theorem 2.13 in Section 7.

\section{The explicit formula and needed sums}

Our starting point for investigating the behavior of low-lying zeros is the explicit formula, which relates sums over zeros to sums over primes. We follow the derivation in [Montgomery and Vaughan 2007] (see also [Iwaniec et al. 2000; Rudnick and Sarnak 1996] and [Davenport 1980; Iwaniec and Kowalski 2004] for all needed results about Dirichlet $L$-functions). We first derive the expansion for Dirichlet characters with fixed conductor $q$, and then extend to $q \in(Q / 2, Q]$. We conclude with some technical estimates that will be of use in proving Theorem 1.2. Here and throughout, we will set $f:=\hat{\eta}$. Note that $\eta$ is real and even, and thus so is the case for $f$, and moreover we have $\hat{f}=\eta$.

\section{A. The explicit formula for fixed q.}

Proposition 3.1 (explicit formula for the family of Dirichlet characters modulo $q$ ). Let $f$ be an even, twice differentiable test function with compact support. Denote the nontrivial zeros of $L(s, \chi)$ by

$$
\rho_{\chi}=\frac{1}{2}+i \gamma_{\chi}
$$


Then the 1-level density $D_{1, q}(\widehat{f})$ equals

$$
\begin{aligned}
\frac{1}{\phi(q)} \sum_{\chi \bmod } \sum_{\gamma_{\chi}} \hat{f}\left(\gamma_{\chi} \frac{\log Q}{2 \pi}\right) & \\
= & \frac{f(0)}{\log Q}\left(\log q-\log \left(8 \pi e^{\gamma}\right)-\sum_{p \mid q} \frac{\log p}{p-1}\right)+\int_{0}^{\infty} \frac{f(0)-f(t)}{Q^{t / 2}-Q^{-t / 2}} d t \\
& -\frac{2}{\log Q} \sum_{\substack{p^{v} \| q \\
p^{e} \equiv 1 \bmod q / p^{v} \\
e, v \geq 1}} \frac{\log p}{\phi\left(p^{v}\right) p^{e / 2}} f\left(\frac{\log p^{e}}{\log Q}\right) \\
& -\frac{2}{\log Q}\left(\sum_{n \equiv 1 \bmod q}-\frac{1}{\phi(q)} \sum_{n}\right) \frac{\Lambda(n)}{n^{1 / 2}} f\left(\frac{\log n}{\log Q}\right)+O\left(\frac{1}{\phi(q)}\right) .
\end{aligned}
$$

Proof. We start with Weil's explicit formula for $L(s, \chi)$, with $\chi \bmod q$ a nonprincipal character (we add the contribution from the principal character later). We can replace $L(s, \chi)$ by $L\left(s, \chi^{*}\right)$ (where $\chi^{*}$ is the primitive character of conductor $q^{*}$ inducing $\chi)$, since these have the same nontrivial zeros. Taking

$$
F(x):=\frac{2 \pi}{\log Q} f\left(\frac{2 \pi x}{\log Q}\right)
$$

in Theorem 12.13 of [Montgomery and Vaughan 2007] (whose conditions are satisfied by our restrictions on $f$ ), we find

$$
\Phi(s)=\hat{f}\left(\frac{\log Q}{2 \pi} \frac{\left(s-\frac{1}{2}\right)}{i}\right),
$$

and

$$
\begin{aligned}
\sum_{\rho_{\chi}} \hat{f}\left(\frac{\log Q}{2 \pi} \gamma_{\chi}\right)= & \frac{f(0)}{\log Q}\left(\log \frac{q^{*}}{\pi}+\frac{\Gamma^{\prime}}{\Gamma}\left(\frac{1}{4}+\frac{a(\chi)}{2}\right)\right) \\
& -\frac{2}{\log Q} \sum_{n=1}^{\infty} \frac{\Lambda(n) \Re\left(\chi^{*}(n)\right)}{n^{1 / 2}} f\left(\frac{\log n}{\log Q}\right) \\
& +\frac{4 \pi}{\log Q} \int_{0}^{\infty} \frac{e^{-(1+2 a(\chi)) \pi x}}{1-e^{-4 \pi x}}\left(f(0)-f\left(\frac{2 \pi x}{\log Q}\right)\right) d x,
\end{aligned}
$$

where $a(\chi)=0$ for the half of the characters with $\chi(-1)=1$ and 1 for the half with $\chi(-1)=-1$. Making the substitution $t=2 \pi x / \log Q$ in the integral and summing over $\chi \neq \chi_{0}$, we find 


$$
\begin{aligned}
& \sum_{\chi \neq \chi_{0}} \sum_{\gamma_{\chi}} \hat{f}\left(\gamma_{\chi} \frac{\log Q}{2 \pi}\right) \\
&=\frac{f(0)}{\log Q}\left(\sum_{\chi \neq \chi_{0}} \log \left(q^{*} / \pi\right)+\frac{\phi(q)}{2} \frac{\Gamma^{\prime}}{\Gamma}\left(\frac{3}{4}\right)+\frac{\phi(q)}{2} \frac{\Gamma^{\prime}}{\Gamma}\left(\frac{1}{4}\right)\right) \\
&+\phi(q) \int_{0}^{\infty} \frac{Q^{-3 t / 2}+Q^{-t / 2}}{1-Q^{-2 t}}(f(0)-f(t)) d t \\
& \quad-\frac{2}{\log Q}\left(\phi(q) \sum_{n \equiv 1 \bmod q}-\sum_{n}\right) \frac{\Lambda(n)}{n^{1 / 2}} f\left(\frac{\log n}{\log Q}\right) \\
& \quad-\frac{2}{\log Q} \sum_{\chi \neq \chi_{0}} \sum_{n} \frac{\Lambda(n) \Re\left(\chi^{*}(n)-\chi(n)\right)}{n^{1 / 2}} f\left(\frac{\log n}{\log Q}\right) \\
&+O(1) .
\end{aligned}
$$

To get (3-3) from (3-2) we added zero by writing $\chi^{*}(n)$ as $\left(\chi^{*}(n)-\chi(n)\right)+\chi(n)$. Summing $\chi(n)$ over all $\chi \bmod q$ gives $\phi(q)$ if $n \equiv 1 \bmod q$ and 0 otherwise; as our sum omits the principal character, the sum of $\chi(n)$ over the nonprincipal characters yields the sum on the third line above. We also replaced $(\phi(q)-1) / 2$ by $\phi(q) / 2$ in the first term, hence the $O(1)$.

We use [Fiorilli and Martin 2013, Proposition 3.3] for the first term (which involves the sum over the conductor of the inducing character). We then use the duplication formula of the digamma function $\psi(z)=\Gamma^{\prime}(z) / \Gamma(z)$ to simplify the next two terms, namely $\psi\left(\frac{1}{4}\right)+\psi\left(\frac{3}{4}\right)$. As $\psi\left(\frac{1}{2}\right)=-\gamma-2 \ln 2$ (Equation 6.3 .3 of [Abramowitz and Stegun 1972]) and $\psi(2 z)=\frac{1}{2} \psi(z)+\frac{1}{2} \psi\left(z+\frac{1}{2}\right)+\ln 2$ (Equation 6.3.8, [ibid.]), setting $z=\frac{1}{4}$ yields $\psi\left(\frac{1}{4}\right)+\psi\left(\frac{3}{4}\right)=-2 \gamma-6 \ln 2$. We keep the next two terms as they are, and then apply [Fiorilli and Martin 2013, Proposition 3.4] (with $r=1$ ) for the last term, obtaining that it equals

$$
-\frac{2}{\log Q} \sum_{n} \frac{\Lambda(n)}{n^{1 / 2}} f\left(\frac{\log n}{\log Q}\right) \Re\left(\sum_{\chi \neq \chi_{0}}\left(\chi^{*}(n)-\chi(n)\right)\right) .
$$

Writing $n=p^{e}$, this term is zero unless $p \mid q$. If $p \mid q$, then it is zero unless $p^{e} \equiv 1 \bmod q / p^{v}$, where $v \geq 1$ is the largest $v$ such that $p^{v} \mid q$. Therefore this term equals

$$
-\frac{2}{\log Q} \sum_{p} \sum_{\substack{p^{v} \| q \\ p^{e} \equiv 1 \bmod q / p^{v} \\ e, \nu \geq 1}} \frac{\Lambda\left(p^{e}\right)}{\phi\left(p^{v}\right) p^{e / 2}} f\left(\frac{\log p^{e}}{\log Q}\right) .
$$

Combining this with some elementary algebra yields 


$$
\begin{aligned}
& \frac{1}{\phi(q)} \sum_{\chi \neq \chi_{0}} \sum_{\gamma_{\chi}} \hat{f}\left(\gamma_{\chi} \frac{\log Q}{2 \pi}\right) \\
& =\frac{f(0)}{\log Q}\left(\log q-\log \left(8 \pi e^{\gamma}\right)-\sum_{p \mid q} \frac{\log p}{p-1}\right)+\int_{0}^{\infty} \frac{f(0)-f(t)}{Q^{t / 2}-Q^{-t / 2}} d t \\
& -\frac{2}{\log Q}\left(\sum_{n \equiv 1 \bmod q}-\frac{1}{\phi(q)} \sum_{n}\right) \frac{\Lambda(n)}{n^{1 / 2}} f\left(\frac{\log n}{\log Q}\right)
\end{aligned}
$$

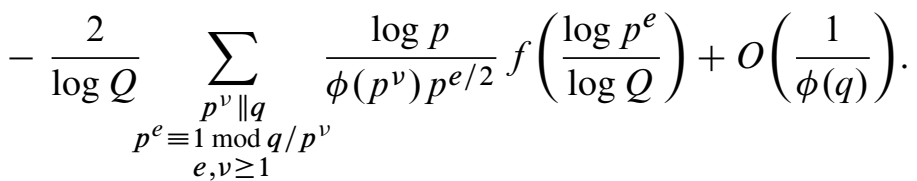

Finally, since the nontrivial zeros of $L\left(s, \chi_{0}\right)$ coincide with those of $\zeta(s)$, the difference between the left-hand side of (3-1) and that of (3-6) is

$$
\frac{1}{\phi(q)} \sum_{\gamma_{\zeta}} \hat{f}\left(\gamma_{\zeta} \frac{\log Q}{2 \pi}\right) \ll \frac{1}{\phi(q)}
$$

(since $f$ is twice continuously differentiable, $\left.\widehat{f}(y) \ll 1 / y^{2}\right)$, completing the proof. ${ }^{6}$

3B. The averaged explicit formula for $q \in(Q / 2, Q]$. We now average the explicit formula for $D_{1 ; q}(\widehat{f})$ (Proposition 3.1) over $q \in(Q / 2, Q]$. We concentrate on deriving useful expansions, which we then analyze in later sections when we determine the allowable support.

Proposition 3.2 (explicit formula for the averaged family of Dirichlet characters modulo $q$ ). The averaged 1-level density, $D_{1 ; Q / 2, Q}(\widehat{f})$, equals

$$
\begin{aligned}
\frac{1}{Q / 2} \sum_{Q / 2<q \leq Q} D_{1 ; q}(\hat{f}) \\
=\frac{f(0)}{\log Q}\left(\log Q-1-\gamma-\log (4 \pi)-\sum_{p} \frac{\log p}{p(p-1)}\right)+\int_{0}^{\infty} \frac{f(0)-f(t)}{Q^{t / 2}-Q^{-t / 2}} d t \\
\quad+\frac{2}{Q / 2} \sum_{Q / 2<q \leq Q} \int_{0}^{\infty}\left(\frac{f(u)}{2}-\frac{f^{\prime}(u)}{\log Q}\right) \frac{\psi\left(Q^{u} ; q, 1\right)-\psi\left(Q^{u}\right) / \phi(q)}{Q^{u / 2}} d u \\
\quad+O\left(\frac{1}{Q}\right) .
\end{aligned}
$$

${ }^{6}$ While the explicit formula for $\zeta(s)$ has a term arising from its pole at $s=1$, that term does not matter here as it is insignificant upon division by the family's size. 


\section{Setting}

$$
\psi_{2}(x ; q, a):=\sum_{\substack{n \leq x \\ n \equiv a \bmod q}} \Lambda(n)\left(1-\frac{n}{x}\right), \quad \psi_{2}(x):=\sum_{n \leq x} \Lambda(n)\left(1-\frac{n}{x}\right),
$$

the last integral in (3-8) may be replaced with

$$
-2 \int_{0}^{\infty}\left(\frac{3 f(u)}{4}-\frac{2 f^{\prime}(u)}{\log Q}+\frac{f^{\prime \prime}(u)}{(\log Q)^{2}}\right) \frac{\psi_{2}\left(Q^{u} ; q, 1\right)-\psi_{2}\left(Q^{u}\right) / \phi(q)}{Q^{u / 2}} d u .
$$

Proof. The main term in the expansion of $D_{1 ; q}(\widehat{f})$ from Proposition 3.1 is

$$
T_{1}(q):=\frac{f(0)}{\log Q}\left(\log q-\log \left(8 \pi e^{\gamma}\right)-\sum_{p \mid q} \frac{\log p}{p-1}\right) .
$$

Using the antiderivative of $\log x$ is $x \log x-x$, one easily finds its average over $Q / 2<q \leq Q$ is

$$
\begin{aligned}
& \frac{1}{Q / 2} \sum_{Q<q \leq 2 Q} T_{1}(q) \\
& \quad=\frac{f(0)}{\log Q}\left(\log Q-1-\gamma-\log (4 \pi)-\sum_{p} \frac{\log p}{p(p-1)}\right)+O\left(\frac{1}{Q}\right) .
\end{aligned}
$$

We now turn to the lower-order term

$$
T_{2}(q):=-\frac{2}{\log Q} \sum_{\substack{p^{v} \| q \\ p^{e} \equiv 1 \bmod q / p^{v} \\ e, \nu \geq 1}} \frac{\log p}{\phi\left(p^{v}\right) p^{e / 2}} f\left(\frac{\log p^{e}}{\log Q}\right) .
$$

Before determining its average behavior, we note that its size can vary greatly with $q$. It is very small for prime $q$ (so $v=1$ and $p=q$ in the sum), since

$$
T_{2}(q) \ll \frac{1}{\log Q} \sum_{e \geq 1} \frac{\log q}{\phi(q) q^{e / 2}} \ll \frac{1}{(q-1)\left(q^{1 / 2}-1\right)} ;
$$

however, it can be as large as $C /(\sqrt{q} \log Q)$ for other values of $q$ (such as $q=$ $\left.2\left(2^{e}-1\right)\right)$. This is, more or less, as large as it can get, since for general $q$ we have

$$
T_{2}(q) \ll \frac{1}{\log Q} \sum_{\substack{p^{v} \| q \\ e, v \geq 1 \\ p^{e} \leq Q^{\sigma}}} \frac{\log p}{\phi\left(p^{v}\right)\left(q / p^{v}\right)^{1 / 2}} \ll \frac{(\log q)^{1 / 2}}{q^{1 / 2} \log \log q} .
$$


On average, however, $T_{2}(q)$ is very small:

$$
\begin{aligned}
& \frac{1}{Q / 2} \sum_{Q / 2<q \leq Q} T_{2}(q) \ll \frac{1}{Q} \sum_{\substack { Q<2<q \leq Q \\
\begin{subarray}{c}{p^{\nu} \| q \\
p^{e} \equiv 1 \bmod q / p^{\nu} \\
e, \nu \geq 1{ Q < 2 < q \leq Q \\
\begin{subarray} { c } { p ^ { \nu } \| q \\
p ^ { e } \equiv 1 \operatorname { m o d } q / p ^ { \nu } \\
e , \nu \geq 1 } }\end{subarray}} \frac{\log p}{p^{\nu+e / 2}} \\
& \ll \frac{1}{Q} \sum_{\substack{p^{v} \\
v, e \geq 1}} \frac{\log p}{p^{v+e / 2}} \sum_{\substack{q \leq Q \\
p^{v} \mid q}} 1 \ll \frac{1}{Q} \sum_{\substack{p^{v} \\
v, e \geq 1}} \frac{\log p}{p^{v+e / 2}} \tau\left(p^{e}-1\right) \\
& \frac{q}{p^{v}} \mid p^{e}-1 \\
& \ll_{\epsilon} \frac{1}{Q} \sum_{\substack{p^{v} \\
\nu, e \geq 1}} \frac{\log p}{p^{\nu+(1-\epsilon) e / 2}} \ll \frac{1}{Q} \sum_{p} \frac{\log p}{p^{3 / 2-\epsilon / 2}} \ll \frac{1}{Q} \text {. }
\end{aligned}
$$

While we will not rewrite the next lower order term, it is instructive to determine its size. Set

$$
T_{3}(q):=\int_{0}^{\infty} \frac{f(0)-f(t)}{Q^{t / 2}-Q^{-t / 2}} d t
$$

Letting $t=2 \pi x / \log Q$, we find

$$
T_{3}(q)=\frac{2 \pi}{\log Q} \int_{0}^{\infty} \frac{f(0)-f(2 \pi x / \log Q)}{2 \sinh (\pi x)} d x .
$$

Since $f$ is twice differentiable with compact support, $|f(0)-f(x)| \ll|x|$, thus

$$
T_{3}(q) \ll \frac{2 \pi}{\log Q} \int_{0}^{\infty} \frac{x}{2 \sinh (\pi x)} d x=\frac{\pi}{4 \log Q} .
$$

As

$$
\int_{0}^{\infty} \frac{x^{k} d x}{\sinh (\pi x)}=\frac{2^{k+1}-1}{2^{k} \pi^{k+1}} \Gamma(k+1) \zeta(k+1),
$$

if $f$ has a Taylor series of order $K+1$ we have

$$
T_{3}(q)=\sum_{k=1}^{K} \frac{\left(2^{k+1}-1\right) \zeta(k+1) f^{(k)}(0)}{\log ^{k+1} Q}+O\left(\frac{1}{\log ^{K+1} Q}\right)
$$

If the Taylor coefficients of $f$ decay very fast, we can even make our bounds uniform and get an error term smaller than a negative power of $Q$.

The remaining term from Proposition 3.1 is the most important, and controls the allowable support. The arithmetic lives here, as this term involves primes in 
arithmetic progressions. It is

$$
\begin{aligned}
T_{4}(q) & :=-\frac{2}{\log Q}\left(\sum_{n \equiv 1 \bmod q}-\frac{1}{\phi(q)} \sum_{n}\right) \frac{\Lambda(n)}{n^{1 / 2}} f\left(\frac{\log n}{\log Q}\right) \\
& =-\frac{2}{\log Q} \int_{1}^{\infty} t^{-1 / 2} f\left(\frac{\log t}{\log Q}\right) d\left(\psi(t ; q, 1)-\frac{\psi(t)}{\phi(q)}\right) \\
& =\frac{2}{\log Q} \int_{1}^{\infty} \frac{\frac{1}{2} f\left(\frac{\log t}{\log Q}\right)-\frac{1}{\log Q} f^{\prime}\left(\frac{\log t}{\log Q}\right)}{t^{3 / 2}}\left(\psi(t ; q, 1)-\frac{\psi(t)}{\phi(q)}\right) d t .
\end{aligned}
$$

The claim in the proposition follows by changing variables by setting $t=Q^{u}$; specifically, the final integral is

$$
T_{4}(q)=2 \int_{0}^{\infty}\left(\frac{f(u)}{2}-\frac{f^{\prime}(u)}{\log Q}\right) \frac{\psi\left(Q^{u} ; q, 1\right)-\psi\left(Q^{u}\right) / \phi(q)}{Q^{u / 2}} d u .
$$

We give an alternative expansion for the final integral. This expansion involves a smoothed sum of $\Lambda(n)$, which will be technically easier to analyze when we turn to determining the allowable support under Montgomery's hypothesis (Theorem 2.13(1)). Recall

$$
\psi_{2}(x ; q, a):=\sum_{\substack{n \leq x \\ n \equiv a \bmod q}} \Lambda(n)\left(1-\frac{n}{x}\right), \quad \psi_{2}(x):=\sum_{n \leq x} \Lambda(n)\left(1-\frac{n}{x}\right),
$$

We integrate by parts in (3-22). Since

$$
\begin{aligned}
\int_{1}^{x}(\psi(t ; q, 1)- & \left.\frac{\psi(t)}{\phi(q)}\right) d t \\
& =\int_{1}^{x}\left(\sum_{\substack{n \leq t \\
n \equiv 1 \bmod q}} \Lambda(n)-\frac{1}{\phi(q)} \sum_{n \leq t} \Lambda(n)\right) d t \\
& =\sum_{\substack{n \leq x \\
n \equiv 1 \bmod q}} \Lambda(n) \int_{n}^{x} d t-\frac{1}{\phi(q)} \sum_{n \leq x} \Lambda(n) \int_{n}^{x} d t \\
& =x\left(\sum_{\substack{n \leq x \\
n \equiv 1 \bmod q}} \Lambda(n)\left(1-\frac{n}{x}\right)-\frac{1}{\phi(q)} \sum_{n \leq x} \Lambda(n)\left(1-\frac{n}{x}\right)\right),
\end{aligned}
$$

we find

$$
T_{4}(q)=-2 \int_{0}^{\infty}\left(\frac{3 f(u)}{4}-\frac{2 f^{\prime}(u)}{\log Q}+\frac{f^{\prime \prime}(u)}{(\log Q)^{2}}\right) \frac{\psi_{2}\left(Q^{u} ; q, 1\right)-\psi_{2}\left(Q^{u}\right) / \phi(q)}{Q^{u / 2}} d u,
$$

completing the proof. 
Remark 3.3. It will be convenient later that in the averaged case $\psi$ and $\psi_{2}$ are both evaluated at $\left(Q^{u} ; q, 1\right)$ and not $\left(q^{u} ; q, 1\right)$; this is because we are rescaling all $L$-function zeros by the same quantity (a global rescaling instead of a local rescaling).

3C. Technical estimates. In the proof of Theorem 2.6, we need the following estimation of a weighted sum of the reciprocal of the totient function.

Lemma 3.4. Let $\phi$ be Euler's totient function. We have

$$
\begin{aligned}
\sum_{r \leq R} \frac{1}{\phi(r)}\left(R^{1 / 2}+\frac{r}{R^{1 / 2}}-2 r^{1 / 2}\right) & \\
& =D_{1} R^{1 / 2} \log R+D_{2} R^{1 / 2}+D_{3}+O\left(\frac{\log R}{R^{1 / 2}}\right)
\end{aligned}
$$

where

$$
\begin{gathered}
D_{1}:=\frac{\zeta(2) \zeta(3)}{\zeta(6)}, \quad D_{2}:=D_{1}\left(\gamma-3-\sum_{p} \frac{\log p}{p^{2}-p+1}\right), \\
D_{3}:=-2 \zeta\left(i \frac{1}{2} i\right) i \prod_{p}\left(i 1+\frac{1}{(p-1) p^{1 / 2}}\right) .
\end{gathered}
$$

More generally, if $P(u):=\sum_{i=0}^{d} a_{i} u^{i}$ is a polynomial of degree $d$ and of norm

$$
\|P\|:=\max _{i}\left|a_{i}\right|
$$

then

$$
\begin{aligned}
& \sum_{r \leq R} \frac{1}{\phi(r)} \int_{\frac{\log r}{\log R}}^{1} P(u)\left(R^{u / 2}-\frac{r}{R^{u / 2}}\right) d u \\
&=E_{1} \log R \int_{-\infty}^{1} R^{u / 2} u P(u) d u+E_{2} \int_{-\infty}^{1} R^{u / 2} P(u) d u \\
& \\
&+\sum_{j=1}^{d+1} \frac{F_{j}(P)}{(\log R)^{j}}+O_{d}\left(R^{-1 / 2}\|P\|\right),
\end{aligned}
$$

where

$$
E_{1}:=\frac{\zeta(2) \zeta(3)}{\zeta(6)}, \quad E_{2}:=E_{1}\left(\gamma-1-\sum_{p} \frac{\log p}{p^{2}-p+1}\right),
$$

and the $F_{j}(P)$ are constants depending on $P$ which can be computed explicitly. 
For example,

$$
\begin{aligned}
F_{1}(P)=-4 \zeta\left(\frac{1}{2}\right) \prod_{p}\left(1+\frac{1}{(p-1) p^{1 / 2}}\right) \sum_{i=0}^{d}(-1)^{i} P^{(i)}(1) \\
F_{2}(P)=-4 \zeta\left(\frac{1}{2}\right) \prod_{p}\left(1+\frac{1}{(p-1) p^{1 / 2}}\right) \\
\times\left(\frac{\zeta^{\prime}}{\zeta}\left(\frac{1}{2}\right)-\sum_{p} \frac{\log p}{(p-1) p^{1 / 2}+1}\right) \sum_{i=1}^{d}(-1)^{i} P^{(i)}(1) .
\end{aligned}
$$

Finally,

$$
\begin{gathered}
\sum_{r \leq R} \frac{1}{\phi(r)} \int_{\frac{\log (r / 2)}{\log (R / 2)}}^{1} P(u)\left((R / 2)^{u / 2}-\frac{r}{2(R / 2)^{u / 2}}\right) d u \\
=E_{1} \log (R / 2) \int_{-\infty}^{1}(R / 2)^{u / 2} u P(u) d u \\
+\left(E_{2}+E_{1} \log 2\right) \int_{-\infty}^{1}(R / 2)^{u / 2} P(u) d u \\
+\sum_{j=1}^{d+1} \frac{F_{j}^{(2)}(P)}{(\log (R / 2))^{j}}+O_{d}\left(R^{-1 / 2}\|P\|\right)
\end{gathered}
$$

where the first two constants are given by

$$
\begin{aligned}
F_{1}^{(2)}(P):= & \frac{F_{1}(P)}{\sqrt{2}} \\
F_{2}^{(2)}(P):= & -2 \sqrt{2} \zeta\left(\frac{1}{2}\right) \prod_{p}\left(1+\frac{1}{(p-1) p^{1 / 2}}\right) \\
& \times\left(\frac{\zeta^{\prime}}{\zeta}\left(\frac{1}{2}\right)-\sum_{p} \frac{\log p}{(p-1) p^{1 / 2}+1}+\log 2\right) \sum_{i=1}^{d}(-1)^{i} P^{(i)}(1) .
\end{aligned}
$$

Remark 3.5. It is possible to improve the estimates in (3-27), (3-30) and (3-33) to ones with an error term of $O_{\epsilon, d}\left(R^{-5 / 4+\epsilon}\|P\|\right)$; however, this is not needed for our purposes.

Proof. By Mellin inversion, for $c \geq 2$ the left-hand side of (3-27) equals

$$
\begin{aligned}
\frac{1}{2 \pi i} \int_{\Re(s)=c} Z(s)\left(\frac{R^{s+1 / 2}}{s}+\right. & \left.\frac{R^{s+1 / 2}}{s+1}-2 \frac{R^{s+1 / 2}}{s+\frac{1}{2}}\right) d s \\
& =\frac{1}{2 \pi i} \int_{\Re(s)=c} Z(s) \frac{R^{s+1 / 2}}{2 s\left(s+\frac{1}{2}\right)(s+1)} d s,
\end{aligned}
$$


where

$$
Z(s):=\sum_{n \geq 1} \frac{1}{n^{s} \phi(n)}
$$

Taking Euler products,

$$
Z(s)=\zeta(s+1) \zeta(s+2) Z_{2}(s)
$$

where

$$
Z_{2}(s):=\prod_{p}\left(1+\frac{1}{p(p-1)}\left(\frac{1}{p^{s+1}}-\frac{1}{p^{2 s+2}}\right)\right)
$$

which converges for $\mathfrak{R}(s)>-\frac{3}{2}$. We shift the contour of integration to the left to the line $\mathfrak{R}(s)=-3 / 2+\epsilon$. By a standard residue calculation, we get that (3-35) equals

$$
\begin{aligned}
D_{1} R^{1 / 2} \log R+D_{2} R^{1 / 2} & +D_{3}+D_{4} \frac{\log R}{R^{1 / 2}}+\frac{D_{5}}{R^{1 / 2}} \\
& +\frac{1}{2 \pi i} \int_{\Re(s)=-\frac{3}{2}+\epsilon} Z(s) \frac{R^{s+1 / 2}}{2 s(s+1 / 2)(s+1)} d s
\end{aligned}
$$

for some constants $D_{4}$ and $D_{5}$. The proof now follows from standard bounds on the zeta function, which show that this integral is $\ll_{\epsilon} R^{-1+\epsilon}$. See the proof of [Fiorilli 2012, Lemma 6.9] for more details.

We now move to (3-30). The Mellin transform in this case is (for $\Re(s)>0$ )

$$
\begin{aligned}
\alpha(s) & :=\int_{0}^{R} r^{s-1} \int_{\frac{\log r}{\log R}}^{1} P(u)\left(R^{u / 2}-\frac{r}{R^{u / 2}}\right) d u d r \\
& =\int_{-\infty}^{1} P(u) \int_{0}^{R^{u}} r^{s-1}\left(R^{u / 2}-\frac{r}{R^{u / 2}}\right) d r d u \\
& =\int_{-\infty}^{1} P(u) \frac{R^{u(s+1 / 2)}}{s(s+1)} d u,
\end{aligned}
$$

which is now defined for $\Re(s)>-\frac{1}{2}$. To meromorphically extend $\alpha(s)$ to the whole complex plane, we integrate by parts $n$ times:

$$
\alpha(s)=\frac{R^{s+1 / 2}}{s(s+1)} \sum_{i=0}^{n} \frac{(-1)^{i} P^{(i)}(1)}{(s+1 / 2)^{i+1}(\log R)^{i+1}},
$$

which is a meromorphic function with poles at the points $s=0,-\frac{1}{2},-1$. The integral we need to compute is

$$
\frac{1}{2 \pi i} \int_{\Re(s)=1} Z(s) \alpha(s) d s .
$$


We remark that

$$
\alpha\left(-\frac{3}{2}+\epsilon+i t\right) \ll_{\epsilon, d} \frac{R^{-1+\epsilon}}{t^{3}}\|P\|,
$$

hence the proof is similar as in the previous case, since by shifting the contour of integration to the left, we have

$$
\frac{1}{2 \pi i} \int_{\mathfrak{R}(s)=1} Z(s) \alpha(s) d s=A+O_{\epsilon, d}\left(R^{-1+\epsilon}\|P\|\right),
$$

where $A$ is the sum of the residues of $Z(s) \alpha(s)$ for $-\frac{3}{2}+\epsilon \leq \Re(s) \leq 2$. Note that if $\beta(s):=s(s+1) \alpha(s)$, then

$$
\beta(0)=\int_{-\infty}^{1} R^{u / 2} P(u) d u, \quad \beta^{\prime}(0)=\log R \int_{-\infty}^{1} R^{u / 2} u P(u) d u,
$$

so the residue at $s=0$ equals

$$
\frac{\zeta(2) \zeta(3)}{\zeta(6)} \beta(0)\left(\frac{\beta^{\prime}}{\beta}(0)+\gamma-1-\sum_{p} \frac{\log p}{p^{2}-p+1}\right) .
$$

For the pole at $s=-\frac{1}{2}$, we need to use the analytic continuation of $\alpha(s)$ provided in (3-41), which shows that this residue equals

$$
\sum_{j=1}^{n+1} \frac{F_{j}(P)}{(\log R)^{j}}
$$

where the $F_{j}(P)$ are constants depending on $P$ which can be computed explicitly. For example,

$$
\begin{aligned}
F_{1}(P)=-4 \zeta\left(\frac{1}{2}\right) & \prod_{p}\left(1+\frac{1}{(p-1) p^{1 / 2}}\right) \sum_{i=0}^{d}(-1)^{i} P^{(i)}(1) \\
F_{2}(P)=-4 \zeta\left(\frac{1}{2}\right) & \prod_{p}\left(1+\frac{1}{(p-1) p^{1 / 2}}\right) \\
& \times\left(\frac{\zeta^{\prime}}{\zeta}\left(\frac{1}{2}\right)-\sum_{p} \frac{\log p}{(p-1) p^{1 / 2}+1}\right) \sum_{i=1}^{d}(-1)^{i} P^{(i)}(1) .
\end{aligned}
$$

Moreover, $F_{i}(P) \ll_{d}\|P\|$ for all $i$.

At $s=-1$, we have a double pole with residue

$$
R^{-1 / 2} \sum_{j=0}^{n+1} \frac{G_{j}(P)}{(\log R)^{j}}
$$

for some constants $G_{j}(P) \ll_{d}\|P\|$, hence the proof of (3-30) is complete. 
For the proof of (3-33), we proceed in the same way, noting that the Mellin transform is

$$
\alpha_{2}(s)=\frac{2^{s}}{s(s+1)} \int_{-\infty}^{1} P(u)(R / 2)^{u(s+1 / 2)} d u
$$

which completes the proof of Lemma 3.4.

\section{Unconditional results (Theorems 2.1 and 2.3)}

Using the expansion for the 1-level density $D_{1, q}(\widehat{f})$ and the averaged 1-level density $D_{1 ; Q / 2, Q}(\widehat{f})$ from Propositions 3.1 and 3.2, we prove our unconditional results.

Proof of Theorem 2.1. We start from Proposition 3.1. The only term of (3-1) we need to understand is the last one (the "prime sum"), which is given by

$$
T_{4}(q):=2 \int_{0}^{1}\left(\frac{f(u)}{2}-\frac{f^{\prime}(u)}{\log q}\right) \frac{\psi\left(q^{u} ; q, 1\right)-\psi\left(q^{u}\right) / \phi(q)}{q^{u / 2}} d u .
$$

(We used that the support of $f$ is contained in $[-1,1]$ and we made the substitution $t=q^{u}$.) However, since there are no integers congruent to $1 \bmod q$ in the interval $\left[2, q^{u}\right]$ when $u \leq 1$ (this is also true when $q^{u}$ is replaced by $Q^{u}$, with $Q / 2<q \leq Q$ ), the $\psi\left(q^{u} ; q, 1\right)$ term equals zero. By the Prime Number Theorem there is an absolute, computable constant $c>0$ such that

$$
\begin{aligned}
T_{4}(q)=-2 \int_{0}^{1}\left(\frac{f(u)}{2}-\frac{f^{\prime}(u)}{\log q}\right) \frac{\psi\left(q^{u}\right)}{q^{u / 2} \phi(q)} d u & \\
=-\frac{2}{\phi(q)} \int_{0}^{1} q^{u / 2}\left(\frac{f(u)}{2}-\frac{f^{\prime}(u)}{\log q}\right) d u & \quad+O\left(\frac{1}{\phi(q)} \int_{0}^{\sigma} \frac{q^{u / 2}}{e^{c \sqrt{u \log q}}} d u\right),
\end{aligned}
$$

and the error term is

$$
\ll \frac{q^{\sigma / 4}}{\phi(q)} \int_{0}^{\sigma / 2} e^{-c \sqrt{u \log q}} d u+\frac{e^{-c \sqrt{(\sigma / 2) \log q}}}{\phi(q)} \int_{\sigma / 2}^{\sigma} q^{u / 2} d u \ll \frac{q^{\sigma / 2-1}}{e^{c^{\prime} \sqrt{\sigma \log q}}}
$$

for $q$ large enough (in terms of $\sigma$ ), completing the proof.

Proof of Theorem 2.3. Starting again from (3-1), we have from (3-15)

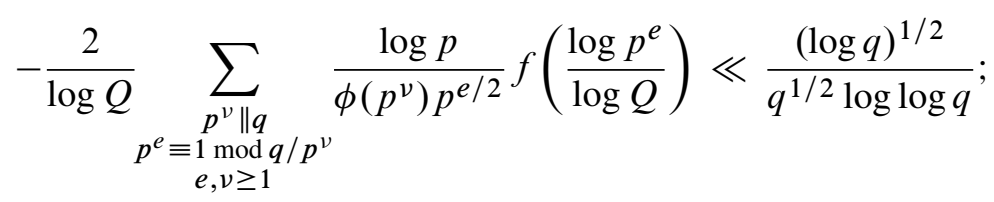


hence this goes in the error term and the only term we need to worry about is the last one.

As our support exceeds $[-1,1]$, the $\psi\left(q^{u} ; q, 1\right)$ no longer trivially vanishes, and the last term is

$$
T_{4}(q)=2 \int_{0}^{2}\left(\frac{f(u)}{2}-\frac{f^{\prime}(u)}{\log q}\right) \frac{\psi\left(q^{u} ; q, 1\right)-\psi\left(q^{u}\right) / \phi(q)}{q^{u / 2}} d u .
$$

In the proof of Theorem 2.1 above we showed that the contribution from the integral where $0 \leq u \leq 1$ is $O\left(q^{-1 / 2}\right)$.

For any fixed $\epsilon>0$, trivial bounds for the region $1 \leq u \leq 1+\epsilon$ yield a contribution that is

$$
\ll \int_{1}^{1+\epsilon}(u \log q) q^{u / 2-1} d u \ll q^{-1 / 2+\epsilon} .
$$

We use the Brun-Titchmarsh Theorem (see [Montgomery and Vaughan 1973]) for the region where $1+\epsilon \leq u \leq 2$, which asserts that for $q<x$,

$$
\pi(x ; q, a) \leq \frac{2 x}{\phi(q) \log (x / q)} .
$$

We first bound the contribution from prime powers as follows. First there are at most $2 e^{\omega(q)}$ residue classes $b \bmod q$ such that $b^{e} \equiv 1 \bmod q$, and so using that $\omega(q) \ll \log q / \log \log q$ we compute

$$
\begin{aligned}
\sum_{e \geq 2} \sum_{\substack{p \leq x^{1 / e} \\
p^{e} \equiv 1 \bmod q}} \log p & \ll \sum_{2 \leq e \leq \frac{2}{\epsilon}} e^{\omega(q)} \max _{b \bmod q}\left(\sum_{\substack{p \leq x^{1 / e} \\
p \equiv b \bmod q}} \log p\right)+\sum_{\frac{2}{\epsilon} \leq e \leq 2 \log x} \sum_{p \leq x^{1 / e}} \log p \\
& \ll \sum_{2 \leq e \leq \frac{2}{\epsilon}} e^{\omega(q)}\left(1+\frac{x^{1 / e}}{q}\right) \log x+\sum_{\frac{2}{\epsilon} \leq e \leq 2 \log x} x^{1 / e} \\
& \ll\left(\frac{2}{\epsilon}\right)^{\omega(q)+1}\left(1+\frac{x^{1 / 2}}{q}\right) \log x+x^{\epsilon / 2} \log x \\
& \ll \epsilon x^{\epsilon}\left(1+\frac{x^{1 / 2}}{q}\right)
\end{aligned}
$$

provided $q$ is large enough in terms of $\epsilon$.

Thus, for $1+\epsilon \leq u \leq 2$, we have

$$
\psi\left(q^{u} ; q, 1\right) \ll_{\epsilon} \frac{q^{u-1} \log \left(q^{u}\right) \log \log q}{(u-1) \log q}+q^{\epsilon}+q^{u / 2-1+\epsilon} \ll_{\epsilon} q^{u-1} \log \log q,
$$

which bounds the integral from $1+\epsilon$ to $\sigma$ by

$$
\ll \int_{1+\epsilon}^{\sigma} q^{u / 2-1} \log \log q d u \ll \frac{\log \log q}{\log q} q^{\sigma / 2-1},
$$


completing the proof.

\section{Results under GRH (Theorems 1.2 and 2.6)}

In this section we assume GRH (but none of the stronger results about the distribution of primes among residue classes) and prove Theorems 1.2 and 2.6. The main ingredient in the proofs are the results of [Fouvry 1985; Bombieri et al. 1986; Friedlander and Granville 1992; Fiorilli 2012]. The following is the needed conditional version.

Theorem 5.1. Assume GRH. Fix an integer $a \neq 0$ and $\epsilon>0$. For $M=M(x) \leq x^{1 / 4}$, we have

$$
\begin{aligned}
& \sum_{\substack{x \\
\frac{x}{2 M}<q \leq \frac{x}{M} \\
(q, a)=1}}\left(\psi(x ; q, a)-\Lambda(a)-\frac{\psi(x)}{\phi(q)}\right) \\
& \quad=\frac{\phi(a)}{a} \frac{x}{2 M} \mu_{0}(a, M)+O_{a, \epsilon}\left(\frac{x}{M^{3 / 2-\epsilon}}+\sqrt{x} M(\log x)^{2}\right),
\end{aligned}
$$

where

$$
\mu_{0}(a, M):= \begin{cases}-\frac{1}{2} \log M-\frac{1}{2} C_{6} & \text { if } a= \pm 1 \\ -\frac{1}{2} \log p & \text { if } a= \pm p^{e} \\ 0 & \text { otherwise }\end{cases}
$$

with

$$
C_{6}:=\log \pi+1+\gamma+\sum_{p} \frac{\log p}{p(p-1)} .
$$

Proof. See [Fiorilli 2012, Remark 1.5]. Note that the restriction $M=o\left(x^{1 / 4} / \log x\right)$ is required for the error term to be negligible compared to the main term, but it can be changed to $M \leq x^{1 / 4}$.

We now proceed to prove Theorems 1.2 and 2.6. Note that by the averaged 1-level density (Proposition 3.2), the proof is completed by analyzing the average of $T_{4}(q)$ :

$$
\begin{aligned}
\frac{1}{Q / 2} & \sum_{Q / 2<q \leq Q} T_{4}(q) \\
= & 2 \int_{0}^{\sigma}\left(\frac{f(u)}{2}-\frac{f^{\prime}(u)}{\log Q}\right) \frac{1}{Q / 2} \sum_{Q / 2<q \leq Q} \frac{\psi\left(Q^{u} ; q, 1\right)-\psi\left(Q^{u}\right) / \phi(q)}{Q^{u / 2}} d u .
\end{aligned}
$$

We break the integral into regions and bound each separately. Going through the proof of Theorem 2.1 and applying GRH, we see that the contribution to the 
integral from $u \in[0,1]$ equals

$$
-\frac{4 \log 2}{Q} \frac{\zeta(2) \zeta(3)}{\zeta(6)} \int_{0}^{1} Q^{u / 2}\left(\frac{f(u)}{2}-\frac{f^{\prime}(u)}{\log Q}\right) d u+O\left(\frac{\log ^{2} Q}{Q}\right) .
$$

We now analyze the three cases of the theorem, corresponding to different support restrictions for our test function.

Proof of Theorem 2.6(2). To prove (2-10), we need to understand the part of the integral in (5-4) with $a \leq u \leq 2$. Arguing as in [Friedlander and Granville 1992] (see also the proof of [Fiorilli 2012, Proposition 6.1]), we have, for $x^{1 / 2} \leq Q \leq x$,

$$
\sum_{Q / 2<q \leq Q}\left(\psi(x ; q, 1)-\frac{\psi(x)}{\phi(q)}\right) \ll Q(\log (x / Q)+1)+\frac{x^{3 / 2}(\log x)^{2}}{Q} .
$$

The basic idea to obtain this last estimate is to write

$$
\sum_{Q / 2<q \leq Q} \psi(x ; q, 1)=\sum_{\substack{n \leq x \\ n-1=q r \\ Q / 2<q \leq Q}} \Lambda(n)
$$

and to turn this into a sum over $r \leq 2(x-1) / Q$ of the function

$$
\psi(x ; r, 1)-\psi(r Q / 2+1 ; r, 1) .
$$

One then applies GRH and estimates the resulting sum over $r$ using estimates on the summatory function of $1 / \phi(r)$. Applying (5-6), the part of the integral in (5-4) with $a \leq u \leq 2$ is

$$
\begin{aligned}
& \ll \int_{a}^{\sigma}\left(Q^{-u / 2}\left(\log \left(Q^{u-1}\right)+1\right)+Q^{u-2}\left(\log \left(Q^{u}\right)\right)^{2}\right) d u \\
& \ll Q^{-a / 2}+Q^{\sigma-2} \log Q,
\end{aligned}
$$

Proof of Theorem 2.6(1). We need to study the part of the integral in (5-4) with $1+\kappa \leq u \leq \frac{3}{2}$. We first see that by (5-7), the part of the integral with $\frac{4}{3} \leq u \leq \frac{3}{2}$ is

$$
\ll Q^{-2 / 3}+Q^{\sigma-2} \log Q
$$

We turn to the part of the integral with $1+\kappa \leq u \leq \frac{4}{3}$. We have by Theorem 5.1 (setting $x:=Q^{u}$ and $M:=Q^{u-1}$ ) that it is 


$$
\begin{aligned}
=2 \int_{1+\kappa}^{4 / 3}\left(\frac{f(u)}{2}-\frac{f^{\prime}(u)}{\log Q}\right) Q^{-u / 2}( & -\frac{1}{2} \log \left(Q^{u-1}\right)-\frac{1}{2} C_{6} \\
& \left.+O_{\epsilon}\left(Q^{\frac{1-u}{2}(1-\epsilon)}+Q^{\frac{3}{2} u-2}\left(\log Q^{u}\right)^{2}\right)\right) d u \\
=-\int_{1+\kappa}^{4 / 3}\left((u-1) \log Q+C_{6}\right) Q^{-u / 2}\left(\frac{f(u)}{2}-\frac{f^{\prime}(u)}{\log Q}\right) d u & \\
& +O_{\epsilon}\left(\frac{Q^{-1 / 2-\kappa(1-\epsilon)}}{\log Q}+Q^{-2 / 3} \log Q\right) ;
\end{aligned}
$$

hence (2-9) holds.

Proof of Theorem 1.2. We now turn to (1-5), with $f$ supported in $\left(-\frac{3}{2}, \frac{3}{2}\right)$. Set

$$
\kappa:=\frac{A \log \log Q}{\log Q}
$$

with $A \geq 1$ a constant. As the big- $O$ constant in (5-9) is independent of $\kappa$, we may use (5-9) to estimate the contribution to (5-4) from $u \in[1+\kappa, 4 / 3]$. This part of the integral contributes

$$
\begin{aligned}
&-\int_{1+\kappa}^{4 / 3}\left((u-1) \log Q+C_{6}\right) Q^{-u / 2}\left(\frac{f(u)}{2}-\frac{f^{\prime}(u)}{\log Q}\right) d u \\
&+O_{\epsilon}\left(\frac{Q^{-1 / 2}}{(\log Q)^{A(1-\epsilon)+1}}\right) \ll \frac{Q^{-1 / 2}}{(\log Q)^{A / 2}} .
\end{aligned}
$$

The part of the integral with $\frac{4}{3} \leq u \leq \frac{3}{2}$ was already shown to be $\ll Q^{-2 / 3}+$ $Q^{\sigma-2} \log Q$, and hence is absorbed into the error term since $\sigma<\frac{3}{2}$.

We now come to the heart of the argument, the part of the integral where $1 \leq u \leq 1+\kappa$. Since $f \in C^{2}(\mathbb{R})$, we have that in our range of $u$, the function $g(u):=\frac{1}{2} f(u)-f^{\prime}(u) / \log Q$ satisfies

$$
\begin{aligned}
g(u) & =\frac{f(1)}{2}+\frac{f^{\prime}(1)}{2}(u-1)+O\left((u-1)^{2}\right)-\frac{f^{\prime}(1)}{\log Q}+O\left(\frac{u-1}{\log Q}\right) \\
& =P(u-1)+O\left(\frac{(\log \log Q)^{2}}{(\log Q)^{2}}\right),
\end{aligned}
$$

where

$$
P(u):=\frac{f(1)}{2}-\frac{f^{\prime}(1)}{\log Q}+\frac{f^{\prime}(1)}{2} u .
$$

At this point, if $f$ were $C^{K}(\mathbb{R})$, we could take its Taylor expansion and get an error of

$$
O_{\epsilon, A}\left((\log \log Q)^{K} /(\log Q)^{K}\right)
$$


We cannot apply Theorem 5.1 directly since the error term is not got enough for moderate values of $M$. Instead, we argue as in the proof of [Fiorilli 2012, Proposition 6.1]. Slightly modifying the proof and using GRH, we get

$$
\begin{aligned}
& \sum_{Q / 2<q \leq Q}\left(\psi(x ; q, a)-\frac{\psi(x)}{\phi(q)}\right) \\
& =x\left(-C_{1}-\sum_{r<\frac{x-1}{Q}} \frac{1}{\phi(r)}\left(1-\frac{r}{x / Q}\right)+\sum_{r<\frac{x-1}{Q / 2}} \frac{1}{\phi(r)}\left(1-\frac{r}{2 x / Q}\right)\right) \\
& +O_{\epsilon}\left(\frac{x^{3 / 2+\epsilon / 2}}{Q}\right),
\end{aligned}
$$

with

$$
C_{1}:=\frac{\zeta(2) \zeta(3)}{\zeta(6)} \log 2
$$

(We used that $\sum_{Q / 2<q \leq Q}=\sum_{Q / 2<q \leq x}-\sum_{Q<q \leq x}$, as in the proof of [Fiorilli 2013, Theorem 4.1*].) The contribution of the error term in (5-12) to the part of the integral in (5-4) with $1 \leq u \leq 1+\kappa$ is (remember $\kappa \log Q=A \log \log Q$ )

$$
\ll \int_{1}^{1+\kappa} \frac{1}{Q / 2} \frac{Q^{3 u / 2+\epsilon u / 2} / Q}{Q^{u / 2}} d u \ll_{\epsilon} Q^{-1+\epsilon} .
$$

Therefore, all that remains to complete the proof of Theorem 1.2 it to estimate the contribution to (5-4) from $u \in[1,1+\kappa]$. Using [Fiorilli 2012, Lemma 5.9] to bound the error in replacing $g(u)$ with $P(u-1)$, we find

$$
\begin{array}{r}
2 \int_{1}^{1+\kappa} g(u) \frac{Q^{u / 2}}{Q / 2}\left(-C_{1}-\sum_{r<\frac{Q^{u}-1}{Q}} \frac{1}{\phi(r)}\left(1-\frac{r}{Q^{u-1}}\right)+\sum_{r<\frac{Q_{-1}}{Q / 2}} \frac{1}{\phi(r)}\left(1-\frac{r}{2 Q^{u-1}}\right)\right) d u \\
=4 \int_{1}^{1+\kappa} P(u-1) Q^{u / 2-1} \\
\left(-C_{1}-\sum_{r \leq \frac{Q^{u}-1}{Q}} \frac{1}{\phi(r)}\left(1-\frac{r}{Q^{u-1}}\right)+\sum_{r \leq \frac{Q_{-1}}{Q / 2}} \frac{1}{\phi(r)}\left(1-\frac{r}{2 Q^{u-1}}\right)\right) d u \\
+O\left(\frac{Q^{-1 / 2}(\log \log Q)^{2}}{(\log Q)^{3}}\right) ; \quad(5-15)
\end{array}
$$

we changed $r<\cdots$ to $r \leq \cdots$ in the sums above, which gives a negligible error term. 
Setting $R:=Q^{\kappa}-1 / Q$, we compute that

$$
\begin{aligned}
\int_{1}^{1+\kappa} & P(u-1) Q^{\frac{u}{2}-1} \sum_{r \leq \frac{Q^{u}-1}{Q}} \frac{1}{\phi(r)}\left(1-\frac{r}{Q^{u-1}}\right) d u \\
= & \frac{1}{Q} \sum_{r \leq Q^{\kappa}-\frac{1}{Q}} \frac{1}{\phi(r)} \int_{1+\frac{\log \left(r+Q^{-1}\right)}{\log Q}}^{1+\kappa} P(u-1)\left(Q^{u / 2}-\frac{r}{Q^{u / 2-1}}\right) d u \\
= & \frac{1}{Q} \sum_{r \leq R} \frac{1}{\phi(r)} \int_{1+\kappa}^{1+\kappa} P(u-1)\left(Q^{u / 2}-\frac{r}{Q^{u / 2-1}}\right) d u+O_{\epsilon}\left(Q^{-3 / 2+\epsilon}\right),
\end{aligned}
$$

the error term coming from the fact that we replaced $\log \left(r+Q^{-1}\right)$ by $\log r$. Performing two changes of variables, we obtain that this is

$$
\begin{aligned}
& =Q^{-1 / 2} \sum_{r \leq R} \frac{1}{\phi(r)} \int_{\kappa \frac{\log r}{\log R}}^{\kappa} P(u)\left(Q^{u / 2}-\frac{r}{Q^{u / 2}}\right) d u+O_{\epsilon}\left(Q^{-3 / 2+\epsilon}\right) \\
& =Q^{-1 / 2} \sum_{r \leq R} \frac{1}{\phi(r)} \int_{\frac{\log r}{\log R}}^{1} \kappa P(\kappa v)\left(R^{v / 2}-\frac{r}{R^{v / 2}}\right) d v+O_{\epsilon}\left(Q^{-3 / 2+\epsilon}\right) .
\end{aligned}
$$

Let

$$
\begin{aligned}
& F_{1}:=-4 \zeta\left(\frac{1}{2}\right) \prod_{p}\left(1+\frac{1}{(p-1) p^{1 / 2}}\right) \\
& F_{2}:=F_{1}\left(\frac{\zeta^{\prime}}{\zeta}\left(\frac{1}{2}\right)-\sum_{p} \frac{\log p}{(p-1) p^{1 / 2}+1}\right) .
\end{aligned}
$$

By Lemma 3.4, we find that (5-16) equals

$$
\begin{aligned}
& \frac{\kappa}{Q^{1 / 2}}\left(E_{1} \log R \int_{-\infty}^{1} R^{u / 2} v P(\kappa v) d v+E_{2} \int_{-\infty}^{1} R^{v / 2} P(\kappa v) d v\right. \\
& \left.+F_{1} \frac{P(\kappa)-\kappa P^{\prime}(\kappa)}{\log R}+F_{2} \frac{-\kappa P^{\prime}(\kappa)}{(\log R)^{2}}+O\left(R^{-1 / 2}\right)\right) \\
& =Q^{-1 / 2}\left(E_{1} \log Q \int_{-\infty}^{\kappa} Q^{u / 2} u P(u) d u+E_{2} \int_{-\infty}^{\kappa} Q^{u / 2} P(u) d u\right. \\
& \left.+F_{1} \frac{\frac{f(1)}{2}-\frac{f^{\prime}(1)}{\log Q}}{\log Q}-F_{2} \frac{f^{\prime}(1)}{2(\log Q)^{2}}+O\left(R^{-1 / 2}\right)\right) .
\end{aligned}
$$


We obtain in an analogous way with $R:=2 Q^{\kappa}-2 / Q$ that

$$
\begin{array}{r}
\int_{1}^{1+\kappa} P(u-1) Q^{u / 2-1} \sum_{r \leq 2 \frac{Q^{u}-1}{Q}} \frac{1}{\phi(r)}\left(1-\frac{r}{2 Q^{u-1}}\right) d u \\
=Q^{-1 / 2} \sum_{r \leq R} \frac{1}{\phi(r)} \int_{\frac{\log (r / 2)}{\log (R / 2)}}^{1} \kappa P(\kappa v)\left((R / 2)^{v / 2}-\frac{r}{2(R / 2)^{v / 2}}\right) d v \\
+O_{\epsilon}\left(Q^{-3 / 2+\epsilon}\right),
\end{array}
$$

which by Lemma 3.4 is

$$
\begin{aligned}
& =\frac{\kappa}{Q^{1 / 2}}\left(E_{1} \log (R / 2) \int_{-\infty}^{1}(R / 2)^{v / 2} v P(\kappa v) d v\right. \\
& +\left(E_{2}+E_{1} \log 2\right) \int_{-\infty}^{1}(R / 2)^{v / 2} P(\kappa v) d v \\
& \left.\quad+\sum_{j=1}^{n} \frac{F_{j}^{(2)}}{(\log (R / 2))^{j}}+O\left(R^{-1 / 2}\right)\right) \\
& =Q^{-1 / 2}\left(E_{1} \log Q \int_{-\infty}^{\kappa} Q^{u / 2} u P(u) d u\right. \\
& +\left(E_{2}+E_{1} \log 2\right) \int_{-\infty}^{\kappa} Q^{u / 2} P(u) d v+\frac{F_{1}}{\sqrt{2}} \frac{\frac{f(1)}{2}-\frac{f^{\prime}(1)}{\log Q}}{\log Q} \\
& \left.-\frac{F_{2}+F_{1} \log 2}{\sqrt{2}} \frac{f^{\prime}(1)}{2(\log Q)^{2}}+O\left(R^{-1 / 2}\right)\right) .
\end{aligned}
$$

We now substitute (5-18) and (5-20) in (5-15), to get that (5-15) is (notice the remarkable cancellations)

$$
\begin{gathered}
=-4 C_{1} \int_{1}^{1+\kappa} P(u-1) Q^{u / 2-1} d u+4 E_{1} \log 2 Q^{-1 / 2} \int_{-\infty}^{\kappa} Q^{u / 2} P(u) d u \\
+4 Q^{-1 / 2}\left(-F_{1} \frac{\frac{f(1)}{2}-\frac{f^{\prime}(1)}{\log Q}}{\log Q}+F_{2} \frac{f^{\prime}(1)}{2(\log Q)^{2}}\right. \\
\left.+\frac{F_{1}}{\sqrt{2}} \frac{\frac{f(1)}{2}-\frac{f^{\prime}(1)}{\log Q}}{\log Q}-\frac{F_{2}+F_{1} \log 2}{\sqrt{2}} \frac{f^{\prime}(1)}{2(\log Q)^{2}}\right) \\
+O\left(Q^{-1 / 2} \frac{(\log \log Q)^{2}}{(\log Q)^{3}}+\frac{Q^{-\frac{1}{2}}}{(\log Q)^{A / 2}}\right),
\end{gathered}
$$


which by (3-31) and (5-13) is

$$
\begin{aligned}
=4 & \log 2 \frac{\zeta(2) \zeta(3)}{\zeta(6)} \int_{-\infty}^{1} P(u-1) Q^{u / 2-1} d u \\
+(2-\sqrt{2}) Q^{-1 / 2}\left(-F_{1}\right. & \left.\frac{f(1)}{\log Q}+\left(F_{2}-\frac{\sqrt{2}+4}{3} F_{1}\right) \frac{f^{\prime}(1)}{(\log Q)^{2}}\right) \\
& +O\left(Q^{-1 / 2} \frac{(\log \log Q)^{2}}{(\log Q)^{3}}+\frac{Q^{-1 / 2}}{(\log Q)^{A / 2}}\right) .
\end{aligned}
$$

But, yet another cancellation is coming: we have

$$
\begin{array}{rl}
\int_{-\infty}^{1} P(u-1) Q^{u / 2-1} & d u \\
& =\int_{-\infty}^{1} g(u) Q^{u / 2-1} d u+O\left(Q^{-1 / 2} \frac{(\log \log Q)^{2}}{(\log Q)^{3}}\right),
\end{array}
$$

and so by (5-5) this term cancels (up to the error term $O\left(Q^{-1}\right)$ ) with the part of the integral of $T_{4}(Q)$ with $u \leq 1$ (which is coming from a totally different part of the problem, where there are no primes in arithmetic progressions involved)!

Combining all the terms,

$$
\begin{aligned}
& \frac{1}{Q / 2} \sum_{Q / 2<q \leq Q} T_{4}(Q) \\
& =(2-\sqrt{2}) Q^{-1 / 2}\left(-F_{1} \frac{f(1)}{\log Q}+\left(F_{2}-\frac{\sqrt{2}+4}{3} F_{1}\right) \frac{f^{\prime}(1)}{(\log Q)^{2}}\right) \\
& \quad+O\left(\frac{Q^{-1 / 2}}{(\log Q)^{A / 2}}+Q^{-1 / 2} \frac{(\log \log Q)^{2}}{(\log Q)^{3}}\right) .
\end{aligned}
$$

The proof is completed by taking $A=6$.

\section{Results under de-averaging hypothesis (Theorem 2.8)}

In this section we assume the de-averaging hypothesis (Hypothesis 2.7), which relates the variance in the distribution of primes congruent to 1 to the average variance over all residue classes. Explicitly, we assume (2-13) holds for some $\delta \in(0,1]$, and show how this allows us to compute the main term in the averaged 1-level density, $D_{1 ; Q / 2, Q}(\widehat{f})$, for test functions $f$ supported in $[-4+2 \delta, 4-2 \delta]$. (Remember that this hypothesis is trivially true for $\delta=1$, and expected to hold for any $\delta>0$.)

Proof of Theorem 2.8. Starting from (3-23), we have

$$
T_{4}(q)=2 \int_{0}^{\infty}\left(\frac{f(u)}{2}-\frac{f^{\prime}(u)}{\log Q}\right) \frac{\psi\left(Q^{u} ; q, 1\right)-\psi\left(Q^{u}\right) / \phi(q)}{Q^{u / 2}} d u .
$$


Feeding this into Proposition 3.2, we are left with determining

$$
\begin{aligned}
& \frac{1}{Q / 2} \sum_{Q / 2<q \leq Q} T_{4}(q) \\
& =\frac{1}{Q / 2} \int_{0}^{\sigma}\left(\frac{f(u)}{2}-\frac{f^{\prime}(u)}{\log Q}\right) Q^{-u / 2} \sum_{Q / 2<q \leq Q}\left(\psi\left(Q^{u} ; q, 1\right)-\frac{\psi\left(Q^{u}\right)}{\phi(q)}\right) d u .
\end{aligned}
$$

We have already seen in the proof of Theorem 2.1 that the part of the integral in (6-2) with $0 \leq u \leq 1$ is $O\left(Q^{-1 / 2}\right)$. For the part where $u \geq 1$, the Cauchy-Schwarz inequality shows that its contribution to the integral in (6-2) is

$$
\ll \frac{1}{Q / 2} \int_{1}^{\sigma} Q^{-u / 2}\left|\sum_{Q / 2<q \leq Q}\left(\psi\left(Q^{u} ; q, 1\right)-\frac{\psi\left(Q^{u}\right)}{\phi(q)}\right)^{2}\right|^{1 / 2}\left|\sum_{Q / 2<q \leq Q} 1^{2}\right|^{1 / 2} d u .
$$

Now, by Hypothesis 2.7, this is

$$
\begin{aligned}
& \ll \frac{1}{Q / 2} \int_{1}^{\sigma} Q^{-u / 2} Q^{(\delta-1) / 2} \\
& \quad \times\left(\sum_{Q / 2<q \leq Q} \sum_{\substack{1 \leq a \leq q \\
(a, q)=1}}\left(\psi\left(Q^{u} ; q, a\right)-\frac{\psi\left(Q^{u}\right)}{\phi(q)}\right)^{2}\right)^{1 / 2} Q^{1 / 2} d u .
\end{aligned}
$$

We now use a result in [Goldston and Vaughan 1997], which states that under GRH we have for $1 \leq Q \leq x$ that

$$
\begin{aligned}
& \sum_{q \leq Q} \sum_{\substack{1 \leq a \leq q \\
(a, q)=1}}\left(\psi(x ; q, a)-\frac{\psi(x)}{\phi(q)}\right)^{2} \\
& =Q x \log Q-c x Q+O_{\epsilon}\left(Q^{2}(x / Q)^{1 / 4+\epsilon}+x^{3 / 2}(\log 2 x)^{5 / 2}(\log \log 3 x)^{2}\right),
\end{aligned}
$$

where

$$
c:=\gamma+\log 2 \pi+1+\sum_{p} \frac{\log p}{p(p-1)} .
$$

We now split the range of integration into the two subintervals $1 \leq u \leq 2$ and $2 \leq u \leq \sigma$. In the first range, we have, for $\epsilon>0$ small enough, $u+1 \geq$ $\max \left(\frac{7}{4}+\frac{1}{4} u+\epsilon(u-1), \frac{3}{2} u\right)$, so (6-5) implies that

$$
\sum_{q \leq Q} \sum_{\substack{1 \leq a \leq q \\(a, q)=1}}\left(\psi(x ; q, a)-\frac{\psi(x)}{\phi(q)}\right)^{2} \ll Q x(\log x)^{3}
$$


(which, up to $x^{\epsilon}$, follows from the original result in [Hooley 1975]), so we get that the part of (6-4) with $1 \leq u \leq 2$ is

$$
\ll Q^{\delta / 2-1} \int_{1}^{2} Q^{-u / 2} Q^{(u+1) / 2}(\log Q)^{3 / 2} d u \ll Q^{(\delta-1) / 2}(\log Q)^{3 / 2},
$$

which is $o(1)$ if $\delta<1$.

We now examine the second interval, that is $2 \leq u \leq \sigma$. In this range, (6-5) becomes

$$
\sum_{q \leq Q} \sum_{\substack{1 \leq a \leq q \\(a, q)=1}}\left(\psi(x ; q, a)-\frac{\psi(x)}{\phi(q)}\right)^{2} \ll x^{3 / 2}(\log x)^{5 / 2}(\log \log x)^{2}
$$

(which, up to a factor of $x^{\epsilon}$, follows from Hooley's original result). We thus get that the part of (6-4) with $2 \leq u \leq \sigma$ is

$$
\begin{aligned}
& \ll \frac{Q^{\delta / 2}}{Q / 2} \int_{2}^{\sigma} Q^{-u / 2} Q^{3 u / 4}(u \log Q)^{5 / 4} \log \log \left(Q^{u}\right) d u \\
& \ll Q^{(\sigma+2 \delta) / 4-1}(\log Q)^{1 / 4} \log \log Q .
\end{aligned}
$$

If $\sigma<4-2 \delta$ then the above is $o(1)$, completing the proof.

\section{Results under Montgomery's hypothesis (Theorem 2.13)}

We continue our investigations beyond the GRH, and assume a smoothed version of Montgomery's hypothesis, Hypothesis 2.12. Interestingly, this assumption allows us to compute the main term of the 1-level density, $D_{1 ; q}(\widehat{f})$, for test functions of arbitrarily large (but finite) support. While similar results have been previously observed [Miller and Sarnak 2003], we include a proof both for completeness and because these observations are not in the literature.

Proof of Theorem 2.13. As we are fixing the modulus, we take $Q:=q$. By the explicit formula from Proposition 3.1, we have

$$
\begin{aligned}
D_{1 ; q}(\widehat{f})= & \frac{f(0)}{\log q}\left(\log q-\log \left(8 \pi e^{\gamma}\right)-\sum_{p \mid q} \frac{\log p}{p-1}\right) \\
& +\int_{0}^{\infty} \frac{f(0)-f(t)}{q^{t / 2}-q^{-t / 2}} d t \\
& -\frac{2}{\log q}\left(\sum_{n \equiv 1 \bmod q}-\frac{1}{\phi(q)} \sum_{n}\right) \frac{\Lambda(n)}{n^{1 / 2}} f\left(\frac{\log n}{\log q}\right)+O\left(\frac{1}{\phi(q)}\right) .
\end{aligned}
$$

Let $\sigma:=\sup (\operatorname{supp} f)<\infty$. We proved in Section 4 that the only terms that are not $O(1 / \log q)$ are the leading term $f(0)$ and possibly the prime sum, which we 
now study. We have

$$
T_{4}(q)=2 \int_{0}^{\infty}\left(\frac{f(u)}{2}-\frac{f^{\prime}(u)}{\log q}\right) \frac{\psi\left(q^{u} ; q, 1\right)-\psi\left(q^{u}\right) / \phi(q)}{q^{u / 2}} d u .
$$

In the proof of Theorem 2.1 we determined that the part of the integral with $0 \leq u \leq 1$ is $O\left(q^{-1 / 2}\right)$. From the proof of Theorem 2.3, the part with $1 \leq u \leq 2$ is $O(\log \log q / \log q)$.

Proof of (1). For the rest of the integral, we use Hypothesis 2.12. Note that $u \geq 2$, so $x=q^{u} \geq q^{2}$ with $u \leq \sigma$, hence we can replace $o_{x \rightarrow \infty}$ by $o_{q \rightarrow \infty}$. An integration by parts gives that the rest of the integral is

$$
\begin{aligned}
=0-( & \left.\frac{f(2)}{2}-\frac{f^{\prime}(2)}{\log q}\right) \frac{\psi_{2}\left(q^{2} ; q, 1\right)-\psi_{2}\left(q^{2}\right) / \phi(q)}{q} \\
& \quad-2 \int_{0}^{\infty}\left(\frac{3 f(u)}{4}-\frac{2 f^{\prime}(u)}{\log q}+\frac{f^{\prime \prime}(u)}{(\log q)^{2}}\right) \frac{\psi_{2}\left(q^{u} ; q, 1\right)-\psi_{2}\left(q^{u}\right) / \phi(q)}{q^{u / 2}} d u \\
= & \frac{o(q)}{q}+\int_{2}^{\sigma}\left(|f(u)|+\left|f^{\prime}(u)\right|+\left|f^{\prime \prime}(u)\right|\right) \frac{o\left(q^{u / 2}\right)}{q^{u / 2}} d u=o(1),
\end{aligned}
$$

proving the claim. Note that we are using the smoothed version of the prime sum. Proof of (2). We already know that the part of the integral with $0 \leq u \leq 1$ is $\ll q^{-1 / 2}$. Taking $\epsilon:=\epsilon^{\prime} / \sigma$ in Hypothesis 2.11, the rest of the integral is $O\left(\int_{1}^{\sigma} q^{\epsilon u-\theta} d u\right)$, which is $O\left(q^{\epsilon^{\prime}-\theta}\right)$ and thus negligible if we may take $\theta>0$.

Remark 7.1. Depending on our assumptions about the size of the error term in the distribution of primes in residue classes, we may allow $\sigma$ to grow with $Q$ at various explicit rates.

\section{Acknowledgments}

The authors thank Andrew Granville, Chris Hughes, Jeffrey Lagarias, Zeév Rudnick and Peter Sarnak for many enlightening conversations, and the referee for many helpful suggestions. Fiorilli was supported by an NSERC doctoral, and later on postdoctoral fellowship, as well as NSF grant DMS-0635607, and pursued this work at the Universite de Montréal, the Institute for Advanced Study and the University of Michigan. Miller was partially supported by NSF grants DMS-0600848, DMS0970067 and DMS-1265673.

\section{References}

[Abramowitz and Stegun 1972] M. Abramowitz and I. A. Stegun (editors), Handbook of mathematical functions with formulas, graphs, and mathematical tables, 10th ed., National Bureau of Standards Applied Mathematics Series 55, U.S. Government Printing Office, Washington, D.C., 1972. Zbl 0543.33001 
[Alpoge and Miller 2014] L. Alpoge and S. J. Miller, "Low-lying zeros of Maass form $L$-functions", preprint, 2014. To appear in IMRN.

[Alpoge et al. 2014] L. Alpoge, N. Amersi, G. Iyer, O. Lazareva, S. J. Miller, and L. Zhang, "Maass waveforms and low-lying zeros", preprint, 2014. arXiv 1306.5886

[Bombieri et al. 1986] E. Bombieri, J. B. Friedlander, and H. Iwaniec, "Primes in arithmetic progressions to large moduli”, Acta Math. 156:3-4 (1986), 203-251. MR 88b:11058 Zbl 0588.10042

[Conrey 2001] J. B. Conrey, “L-functions and random matrices”, pp. 331-352 in Mathematics unlimited-2001 and beyond, edited by B. Engquist and W. Schmid, Springer, Berlin, 2001. MR 2002g:11134 Zbl 1048.11071

[Conrey 2007] J. B. Conrey, “The mean-square of Dirichlet $L$-functions”, preprint, 2007. arXiv $0708.2699 \mathrm{v} 1$

[Conrey and Iwaniec 2002] B. Conrey and H. Iwaniec, "Spacing of zeros of Hecke $L$-functions and the class number problem", Acta Arith. 103:3 (2002), 259-312. MR 2003h:11103 Zbl 1007.11051

[Conrey and Snaith 2007] J. B. Conrey and N. C. Snaith, "Applications of the $L$-functions ratios conjectures”, Proc. Lond. Math. Soc. (3) 94:3 (2007), 594-646. MR 2009a:11179 Zbl 1183.11050

[Conrey and Snaith 2008] J. B. Conrey and N. C. Snaith, "Triple correlation of the Riemann zeros", J. Théor. Nombres Bordeaux 20:1 (2008), 61-106. MR 2009f:11109 Zbl 1208.11103

[Conrey et al. 2005a] J. B. Conrey, D. W. Farmer, J. P. Keating, M. O. Rubinstein, and N. C. Snaith, "Integral moments of L-functions", Proc. London Math. Soc. (3) 91:1 (2005), 33-104. MR 2006j:11120 Zbl 1075.11058

[Conrey et al. 2005b] J. B. Conrey, D. W. Farmer, and M. R. Zimbauer, "Howe pairs, supersymmetry, and ratios of random characteristic polynomials for the unitary groups $U_{N}$ ", preprint, 2005. arXiv math-ph/0511024

[Conrey et al. 2008] B. Conrey, D. W. Farmer, and M. R. Zirnbauer, "Autocorrelation of ratios of $L$ functions", Commun. Number Theory Phys. 2:3 (2008), 593-636. MR 2009j:11138 Zbl 1178.11056

[Davenport 1980] H. Davenport, Multiplicative number theory, 2nd ed., Graduate Texts in Mathematics 74, Springer, New York, 1980. MR 82m:10001 Zbl 0453.10002

[David et al. 2013] C. David, D. K. Huynh, and J. Parks, "One-level density of families of elliptic curves and the ratio conjectures", preprint, 2013. arXiv 1309.1027

[Dueñez and Miller 2006] E. Dueñez and S. J. Miller, "The low lying zeros of a GL(4) and a GL(6) family of L-functions", Compos. Math. 142:6 (2006), 1403-1425. MR 2007k:11141 Zbl 1124.11040

[Dueñez and Miller 2009] E. Dueñez and S. J. Miller, "The effect of convolving families of $L$ functions on the underlying group symmetries", Proc. Lond. Math. Soc. (3) 99:3 (2009), 787-820. MR 2010k:11145 Zbl 1244.11079

[Fiorilli 2012] D. Fiorilli, "Residue classes containing an unexpected number of primes", Duke Math. J. 161:15 (2012), 2923-2943. MR 2999316 Zbl 1264.11081

[Fiorilli 2013] D. Fiorilli, "The influence of the first term of an arithmetic progression", Proc. Lond. Math. Soc. (3) 106:4 (2013), 819-858. MR 3056294 Zbl 06170788

[Fiorilli and Martin 2013] D. Fiorilli and G. Martin, "Inequities in the Shanks-Rényi prime number race: An asymptotic formula for the densities", J. Reine Angew. Math. 676 (2013), 121-212. MR 3028758 Zbl 1276.11150

[Firk and Miller 2009] F. W. K. Firk and S. J. Miller, "Nuclei, primes and the random matrix connection", Symmetry 1:1 (2009), 64-105. MR 2012h:11126 
[Fouvry 1985] É. Fouvry, "Sur le problème des diviseurs de Titchmarsh", J. Reine Angew. Math. 357 (1985), 51-76. MR 87b:11090 Zbl 0547.10039

[Fouvry and Iwaniec 2003] E. Fouvry and H. Iwaniec, "Low-lying zeros of dihedral $L$-functions", Duke Math. J. 116:2 (2003), 189-217. MR 2003k:11139 Zbl 1028.11055

[Friedlander and Granville 1992] J. B. Friedlander and A. Granville, "Relevance of the residue class to the abundance of primes", pp. 95-103 in Proceedings of the Amalfi Conference on Analytic Number Theory (Maiori, 1989), edited by E. Bombieri et al., Univ. Salerno, Salerno, 1992. MR 94i:11067 Zbl 0795.11040

[Friedlander et al. 1991] J. Friedlander, A. Granville, A. Hildebrand, and H. Maier, "Oscillation theorems for primes in arithmetic progressions and for sifting functions", J. Amer. Math. Soc. 4:1 (1991), 25-86. MR 92a:11103 Zbl 0724.11040

[Gao 2005] P. Gao, $N$-level density of the low-lying zeros of quadratic Dirichlet L-functions, Ph.D. thesis, University of Michigan, 2005, http://search.proquest.com/docview/305462205.

[Goes et al. 2010] J. Goes, S. Jackson, S. J. Miller, D. Montague, K. Ninsuwan, R. Peckner, and T. Pham, "A unitary test of the ratios conjecture", J. Number Theory 130:10 (2010), 2238-2258. MR 2011g:11162 Zbl 1276.11141

[Goldfeld 1976] D. M. Goldfeld, "The class number of quadratic fields and the conjectures of Birch and Swinnerton-Dyer”, Ann. Scuola Norm. Sup. Pisa Cl. Sci. (4) 3:4 (1976), 624-663. MR 56 \#8529 Zbl 0345.12007

[Goldston and Vaughan 1997] D. A. Goldston and R. C. Vaughan, "On the Montgomery-Hooley asymptotic formula", pp. 117-142 in Sieve methods, exponential sums, and their applications in number theory (Cardiff, 1995), edited by G. R. H. Greaves et al., London Math. Soc. Lecture Note Ser. 237, Cambridge Univ. Press, 1997. MR 99d:11099 Zbl 0929.11034

[Gonek et al. 2007] S. M. Gonek, C. P. Hughes, and J. P. Keating, "A hybrid Euler-Hadamard product for the Riemann zeta function”, Duke Math. J. 136:3 (2007), 507-549. MR 2008e:11100 Zbl 1171.11049

[Gross and Zagier 1986] B. H. Gross and D. B. Zagier, "Heegner points and derivatives of $L$-series", Invent. Math. 84:2 (1986), 225-320. MR 87j:11057 Zbl 0608.14019

[Güloğlu 2005] A. M. Güloğlu, "Low-lying zeroes of symmetric power L-functions", Int. Math. Res. Not. 2005:9 (2005), 517-550. MR 2006g:11180 Zbl 1168.11312

[Hayes 2003] B. Hayes, “The spectrum of Riemannium”, American Scientist 91:4 (2003), 296-300.

[Heath-Brown 1981] D. R. Heath-Brown, "An asymptotic series for the mean value of Dirichlet L-functions”, Comment. Math. Helv. 56:1 (1981), 148-161. MR 83c:10059 Zbl 0457.10020

[Hejhal 1994] D. A. Hejhal, "On the triple correlation of zeros of the zeta function”, Int. Math. Res. Not. 1994:7 (1994), 294-302. MR 96d:11093 Zbl 0813.11048

[Hooley 1975] C. Hooley, "On the Barban-Davenport-Halberstam theorem, I", J. Reine Angew. Math. 274/275 (1975), 206-223. MR 52 \#3090a Zbl 0304.10027

[Hughes and Miller 2007] C. P. Hughes and S. J. Miller, "Low-lying zeros of $L$-functions with orthogonal symmetry”, Duke Math. J. 136:1 (2007), 115-172. MR 2009b:11145 Zbl 1124.11041

[Hughes and Rudnick 2003] C. P. Hughes and Z. Rudnick, "Linear statistics of low-lying zeros of $L$-functions", Q. J. Math. 54:3 (2003), 309-333. MR 2005a:11131 Zbl 1068.11055

[Huynh et al. 2009] D. K. Huynh, J. P. Keating, and N. C. Snaith, "Lower order terms for the one-level density of elliptic curve L-functions", J. Number Theory 129:12 (2009), 2883-2902. MR 2010i:11094 Zbl 1205.11077 
[Huynh et al. 2011] D. K. Huynh, S. J. Miller, and R. Morrison, "An elliptic curve test of the L-functions ratios conjecture", J. Number Theory 131:6 (2011), 1117-1147. MR 2012d:11142 Zbl 1235.11084

[Iwaniec and Kowalski 2004] H. Iwaniec and E. Kowalski, Analytic number theory, American Mathematical Society Colloquium Publications 53, Amer. Math. Soc., Providence, RI, 2004. MR 2005h:11005 Zbl 1059.11001

[Iwaniec et al. 2000] H. Iwaniec, W. Luo, and P. Sarnak, "Low lying zeros of families of $L$-functions", Inst. Hautes Études Sci. Publ. Math. 91 (2000), 55-131. MR 2002h:11081 Zbl 1012.11041

[Katz and Sarnak 1999a] N. M. Katz and P. Sarnak, Random matrices, Frobenius eigenvalues, and monodromy, American Mathematical Society Colloquium Publications 45, Amer. Math. Soc., Providence, RI, 1999. MR 2000b:11070 Zbl 0958.11004

[Katz and Sarnak 1999b] N. M. Katz and P. Sarnak, "Zeroes of zeta functions and symmetry", Bull. Amer. Math. Soc. (N.S.) 36:1 (1999), 1-26. MR 2000f:11114 Zbl 0921.11047

[Keating and Snaith 2000a] J. P. Keating and N. C. Snaith, "Random matrix theory and $\zeta(1 / 2+i t)$ ", Comm. Math. Phys. 214:1 (2000), 57-89. MR 2002c:11107 Zbl 1051.11048

[Keating and Snaith 2000b] J. P. Keating and N. C. Snaith, "Random matrix theory and $L$-functions at $s=1 / 2$ ", Comm. Math. Phys. 214:1 (2000), 91-110. MR 2002c:11108 Zbl 1051.11047

[Keating and Snaith 2003] J. P. Keating and N. C. Snaith, "Random matrices and $L$-functions", $J$. Phys. A 36:12 (2003), 2859-2881. MR 2004d:11090 Zbl 1074.11053

[Miller 2004] S. J. Miller, "One- and two-level densities for rational families of elliptic curves: Evidence for the underlying group symmetries", Compos. Math. 140 (2004), 952-992. MR 2005c:11085 Zbl 1120.11026

[Miller 2008] S. J. Miller, "A symplectic test of the L-functions ratios conjecture", Int. Math. Res. Not. 2008:3 (2008), Art. ID rnm146. MR 2009h:11139 Zbl 1225.11104

[Miller 2009a] S. J. Miller, "Lower order terms in the 1-level density for families of holomorphic cuspidal newforms”, Acta Arith. 137:1 (2009), 51-98. MR 2010f:11146 Zbl 1214.11100

[Miller 2009b] S. J. Miller, "An orthogonal test of the $L$-functions ratios conjecture", Proc. Lond. Math. Soc. (3) 99:2 (2009), 484-520. MR 2011g:11163 Zbl 1170.11027

[Miller and Montague 2011] S. J. Miller and D. Montague, "An orthogonal test of the $L$-functions ratios conjecture, II”, Acta Arith. 146:1 (2011), 53-90. MR 2012k:11139 Zbl 1233.11054

[Miller and Peckner 2012] S. J. Miller and R. Peckner, "Low-lying zeros of number field $L$-functions", J. Number Theory 132:12 (2012), 2866-2891. MR 2965197 Zbl 06097268

[Miller and Sarnak 2003] S. J. Miller and P. Sarnak, personal communication, 2003.

[Montgomery 1970] H. L. Montgomery, "Primes in arithmetic progressions", Michigan Math. J. 17 (1970), 33-39. MR 41 \#1660 Zbl 0209.34804

[Montgomery 1973] H. L. Montgomery, "The pair correlation of zeros of the zeta function", pp. 181-193 in Analytic number theory (St. Louis, MO, 1972), edited by H. G. Diamond, Amer. Math. Soc., Providence, R.I., 1973. MR 49 \#2590 Zbl 0268.10023

[Montgomery and Vaughan 1973] H. L. Montgomery and R. C. Vaughan, "The large sieve", Mathematika 20 (1973), 119-134. MR 51 \#10260 Zbl 0296.10023

[Montgomery and Vaughan 2007] H. L. Montgomery and R. C. Vaughan, Multiplicative number theory, I: Classical theory, Cambridge Studies in Advanced Mathematics 97, Cambridge University Press, 2007. MR 2009b:11001 Zbl 1142.11001

[Odlyzko 1987] A. M. Odlyzko, "On the distribution of spacings between zeros of the zeta function", Math. Comp. 48:177 (1987), 273-308. MR 88d:11082 Zbl 0615.10049 
[Odlyzko 2001] A. M. Odlyzko, “The $10^{22}$-nd zero of the Riemann zeta function”, pp. 139-144 in Dynamical, spectral, and arithmetic zeta functions (San Antonio, TX, 1999), edited by M. L. Lapidus and M. van Frankenhuysen, Contemp. Math. 290, Amer. Math. Soc., Providence, RI, 2001. MR 2003h:11109 Zbl 1022.11042

[Ricotta and Royer 2011] G. Ricotta and E. Royer, "Statistics for low-lying zeros of symmetric power $L$-functions in the level aspect", Forum Math. 23:5 (2011), 969-1028. MR 2012k:11057 Zbl 1264.11080

[Royer 2001] E. Royer, "Petits zéros de fonctions $L$ de formes modulaires", Acta Arith. 99:2 (2001), 147-172. MR 2002g:11063 Zbl 0984.11024

[Rubinstein 2001] M. Rubinstein, "Low-lying zeros of $L$-functions and random matrix theory", Duke Math. J. 109:1 (2001), 147-181. MR 2002f:11114 Zbl 1014.11050

[Rubinstein and Sarnak 1994] M. Rubinstein and P. Sarnak, "Chebyshev's bias", Experiment. Math. 3:3 (1994), 173-197. MR 96d:11099 Zbl 0823.11050

[Rudnick and Sarnak 1996] Z. Rudnick and P. Sarnak, "Zeros of principal $L$-functions and random matrix theory”, Duke Math. J. 81:2 (1996), 269-322. MR 97f:11074 Zbl 0866.11050

[Shin and Templier 2012] S. W. Shin and N. Templier, "Sato-Tate theorem for families and low-lying zeros of automorphic $L$-functions", preprint, 2012. arXiv 1208.1945v2

[Yang 2009] A. Yang, Distribution problems associated to zeta functions and invariant theory, Ph.D. thesis, Princeton University, 2009, http://search.proquest.com/docview/304982142.

[Young 2005] M. P. Young, "Lower-order terms of the 1-level density of families of elliptic curves", Int. Math. Res. Not. 2005:10 (2005), 587-633. MR 2006c:11076 Zbl 1071.11030

[Young 2006] M. P. Young, "Low-lying zeros of families of elliptic curves", J. Amer. Math. Soc. 19:1 (2006), 205-250. MR 2006d:11072 Zbl 1086.11032

Communicated by Peter Sarnak

Received 2013-09-27 Revised 2014-04-02 Accepted 2014-05-24

fiorilli@umich.edu

Department of Mathematics, University of Michigan, 530 Church Street, Ann Arbor, MI 48109, United States

sjm1@williams.edu

Mathematics and Statistics, Williams College, 18 Hoxsey St, Bronfman Science Center, Williamstown, MA 01267, United States 


\section{Algebra \& Number Theory}

msp.org/ant

\section{EDITORS}

MANAGING EDITOR

Bjorn Poonen

Massachusetts Institute of Technology

Cambridge, USA

\author{
EDITORIAL BOARD CHAIR \\ David Eisenbud \\ University of California \\ Berkeley, USA
}

BOARD OF EDITORS

Georgia Benkart

Dave Benson

Richard E. Borcherds

John H. Coates

J-L. Colliot-Thélène

Brian D. Conrad

Hélène Esnault

Hubert Flenner

Edward Frenkel

Andrew Granville

Joseph Gubeladze

Roger Heath-Brown

Craig Huneke

János Kollár

Yuri Manin

Barry Mazur

Philippe Michel

Susan Montgomery
University of Wisconsin, Madison, USA

University of Aberdeen, Scotland

University of California, Berkeley, USA

University of Cambridge, UK

CNRS, Université Paris-Sud, France

University of Michigan, USA

Freie Universität Berlin, Germany

Ruhr-Universität, Germany

University of California, Berkeley, USA

Université de Montréal, Canada

San Francisco State University, USA

Oxford University, UK

University of Virginia, USA

Princeton University, USA

Northwestern University, USA

Harvard University, USA

École Polytechnique Fédérale de Lausanne

University of Southern California, USA
Shigefumi Mori

Raman Parimala

Jonathan Pila

Anand Pillay

Victor Reiner

Peter Sarnak

Joseph H. Silverman

Michael Singer

Vasudevan Srinivas

J. Toby Stafford

Richard Taylor

Ravi Vakil

Michel van den Bergh

Marie-France Vignéras

Kei-Ichi Watanabe

Efim Zelmanov

Shou-Wu Zhang
RIMS, Kyoto University, Japan

Emory University, USA

University of Oxford, UK

University of Notre Dame, USA

University of Minnesota, USA

Princeton University, USA

Brown University, USA

North Carolina State University, USA

Tata Inst. of Fund. Research, India

University of Michigan, USA

Harvard University, USA

Stanford University, USA

Hasselt University, Belgium

Université Paris VII, France

Nihon University, Japan

University of California, San Diego, USA

Princeton University, USA

\section{PRODUCTION}

production@msp.org

Silvio Levy, Scientific Editor

See inside back cover or msp.org/ant for submission instructions.

The subscription price for 2015 is US \$255/year for the electronic version, and $\$ 440 /$ year ( $\$ 55$, if shipping outside the US) for print and electronic. Subscriptions, requests for back issues and changes of subscribers address should be sent to MSP.

Algebra \& Number Theory (ISSN 1944-7833 electronic, 1937-0652 printed) at Mathematical Sciences Publishers, 798 Evans Hall \#3840, c/o University of California, Berkeley, CA 94720-3840 is published continuously online. Periodical rate postage paid at Berkeley, CA 94704, and additional mailing offices.

ANT peer review and production are managed by EditFLOW ${ }^{\circledR}$ from MSP.

\section{PUBLISHED BY}

7 mathematical sciences publishers

nonprofit scientific publishing

http://msp.org/

(C) 2015 Mathematical Sciences Publishers 


\section{Algebra \& Number Theory}

Volume $9 \quad$ No. $1 \quad 2015$

On Previdi's delooping conjecture for $K$-theory

SHo SAITO

Surpassing the ratios conjecture in the 1-level density of Dirichlet $L$-functions

DANIEL FIORILli and STEVEN J. MiLler

Eisenstein Hecke algebras and conjectures in Iwasawa theory

PRESTON WAKE

Adequate groups of low degree

Robert Guralnick, Florian Herzig and Pham HuU TieP

Random matrices, the Cohen-Lenstra heuristics, and roots of unity

DEREK GARTON

Local Beilinson-Tate operators

173

AMNON YEKUTIELI

Categories of abelian varieties over finite fields, I: Abelian varieties over $\mathbb{F}_{p}$ 